\title{
SmartCrawler: An In-pipe Robotic System with Novel Low- frequency Wireless Communication Setup in Water Distribution Systems
}

This paper was downloaded from TechRxiv (https://www.techrxiv.org).

\section{LICENSE}

CC BY 4.0

\section{SUBMISSION DATE / POSTED DATE}

$11-01-2021 / 14-01-2021$

\section{CITATION}

Kazeminasab, Saber; Banks, M.Katherine (2021): SmartCrawler: An In-pipe Robotic System with Novel Lowfrequency Wireless Communication Setup in Water Distribution Systems. TechRxiv. Preprint. https://doi.org/10.36227/techrxiv.13554197.v1

$\mathrm{DOI}$ 


\title{
SmartCrawler: An In-pipe Robotic System with Novel Low-frequency Wireless Communication Setup in Water Distribution Systems
}

\author{
Saber Kazeminasab, Student Member, IEEE, and M. Katherine Banks .
}

\begin{abstract}
Water Distribution Systems (WDS) are critical infrastructures that deliver potable water to residential areas. Water quality monitoring is one of the requirements for utility managers to ensure the health of the water. However, it is challenging to access all parts of the WDS since they are long and comprise different configurations. In this paper, we propose a size-adaptable in-pipe robot so-called "SmartCrawler". We develop two-phase control algorithm that enables reliable motion in different configurations of pipelines. The controller in phase 1 stabilizes the robot in the straight paths and tracks the desired velocity with high-level linear quadratic regulator (LQR) and low-level proportional-integral-derivative (PID) based controllers. The controller in phase 2 enables the robot to have reliable change of direction in the non-straight paths. The performance of the two-phase controller is evaluated with experimental and simulation results. Wireless underground communication is a challenging task for underground applications. To facilitate wireless communication, we propose an active radio frequency identification

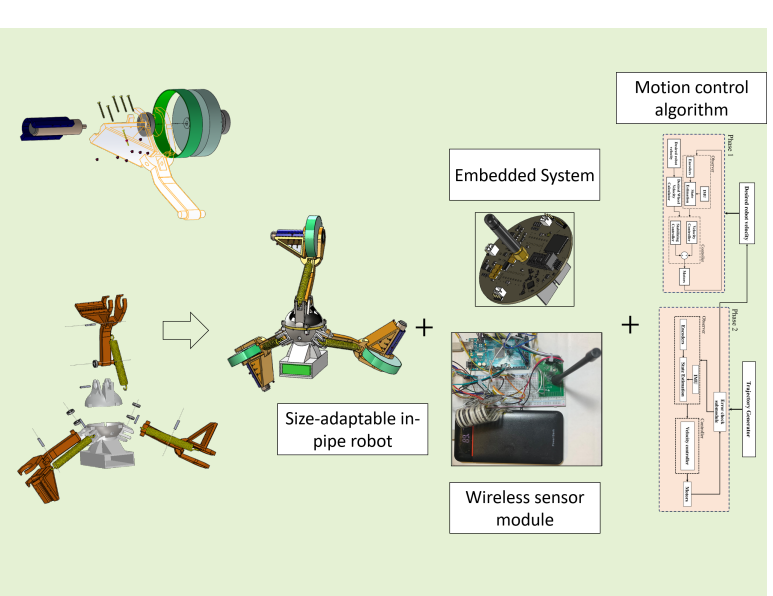
(RFID) based communication working in $434 \mathrm{MHz}$ carrier frequency and evaluate its performance with experimental results. At the end of this work, we design the printed circuit board (PCB) for the SmartCrawler. The simulation and the experimental results prove the proposed robotic system can be used for in-pipe missions where wireless communication is needed to communicate with the robot during operation.
\end{abstract}

Index Terms_-In-pipe robots; Water quality monitoring; Multi-phase control algorithm; Wireless underground communication; Water distribution systems;

\section{INTRODUCTION}

$\mathbf{P}$ IPELINE networks are crucial infrastructures in the oil, gas, and water industries. They carry fluids between cities or countries and are responsible to deliver them to the residential areas. Incidents damage the pipelines which cause leak and result in water loss.

On average, 15 to 25 percent of water loss is reported in the water utility sector in the US . However, in older distribution systems, this amount reaches 40 percent [1]. In Canada, this portion for water loss is 20 percent each year [2] which is

"An earlier versions of this paper were: 1 - presented at the International Conference on Robotics and Automation Engineering (ICRAE, 2020), November 2020 and is in press [38]. The link of the paper can be found at:https://ieeexplore. ieee.org/document/9310805. 2- Accepted at 22nd IEEE International Conference on Industrial Technology (ICIT, 2021) and is in press [37]. The preprint link: https: //arxiv.org/abs/2012.15236

3- Accepted at The 36th ACM/SIGAPP Symposium On Applied Computing (SAC, 2021) and is in press [35]. Preprint link: https : / / arxiv. org/abs/2012.14879

Saber Kazeminasab is with the department of Electrical and Computer Engineering, Texas A\&M University, College Station, Texas, USA,77847 (e-mail: skazeminasab@tamu.edu).

M. Katherine Banks is with the College of Engineering, Texas A\&M University, College Station, Texas, USA,77847 (e-mail: kbanks@tamu.edu). unavoidable and needs to be mitigated. Based on an interview with utility managers in London, the water loss is reported is 30 percent. Besides, the parameters in water in distribution systems should be regularly monitored to ensure the quality of water that is vital to public health [3], [4]. Traditional methods for leak detection and quality monitoring are not efficient due to dependency on user experience and limited coverage of the network [4]-[6]. Mobile sensors are the next option in which they are released in the network in the pipeline and perform the desired task based on the application [7][9]. However, there is a challenge with these sensors as they have passive motion. These small mobile sensors are moved with the flow in the pipeline and due to the passiveness of their motion and complicated configuration of networks, the mobile sensors are not directed to the desired location for retrieval unless that part of the network that is considered for sensor operation is shut down and isolated from the rest of the network. Thus, it is required to design the sensor modules that are automotive during operation in which their motion is independent of the flow inside pipelines. In-pipe robots are promising alternatives for these tasks. They can embed various sensors for doing different tasks (e.g., leak detection, measuring the concentration of target analytes in 
water, inspecting pipelines visually, etc.) in the pipeline.

\section{A. Literature Review}

Most robots are actuated either by pneumatic actuators [10]-[12] or electrical actuators (i.e. DC motors) [13]-[24]. The pneumatic actuated robots need special mechanisms to transform the actuator power to the mechanical components which makes their motion slow during operation. In some robots, new actuators are used to stabilize the robot and move it forward in the network [25]. There are some considerations in robots' operation conditions that need to be addressed in the design of in-pipe robots. Pipes diameter in pipeline networks change in various parts which requires the robots to be sizeadaptable [26]. Pipeline networks are composed of long lines of pipes. These pipes are in someplace buried underground. Hence, the robots need to be able to inspect and cover long distances of pipelines. In this regard, some robots are powered with cable [26]. The short length of cables limits their long pipe inspection. There are a few robots that provide their power from battery [13]. If the robot stocks in pipeline during operation as the battery run out of power, the ground at the location of the stocked robot should be excavated and an access point needs to be created to retrieve the robot. In addition, there are high drag force [27] and pressure in the operation environment that the robot should be able to tackle. Hence, operation conditions (i.e. confined size, high drag force) needs to be considered among battery-powered robots. The pipelines comprise different configurations like bends and T-junctions and the robot should be able to pass through these configurations [28]. Among the robots that are powered with cables, it is possible to transceive data between robot and base station (BS) through wired communication. However, as mentioned, the limited length of cables used for power supply prevents long coverage of pipeline inspection. Besides, based on the feedback from water utility managers, it is not desirable to use wired robots because, in this way, they can take water samples directly from the network through access points. Hence, wireless communication is required for robots for data transmission in this application. However, wireless communication through the harsh and dynamic environment of soil and water is a challenging task [29]. There is high signal attenuation in the medium of water, rock, and soil [29]. Also, the communication channel is highly dynamic as the moisture content of soil affects the path loss as the composition of soil [29]. Based on Friis propagation theory [30], lower frequencies results in higher received signal's power to transmitted signal's power that helps to mitigate signal attenuation at the expense of larger antenna sizes. Magnetic Induction (MI) communication is a solution that mitigates the challenges mentioned for EM waves. In this method, a pair of coils act as a wireless communication link that transmits and receives data based on mutual induction [31]. Sun et al. modeled the MI communication channel and provided a relation for the received signal's power to the transmitted signal's power in [31]. To increase the read range of the MI communication link, they utilized waveguide coils that are parallel to the pipe axis and are mounted on the pipe's circumference [31]. Tan et al. verified the feasibility of waveguides with experimental results in [32]. However, communication efficiency in MI highly depends on the direction of the transmitter and receiver coils. They need to be parallel and if there is a misalignment angle between their normal axes, the efficiency of the communication link drops, dramatically [33]. To address this challenge, Omni-directional coil antennas are considered [34]. However, the size of these antennas limits their applicability for confined spaces in the pipeline. Hence, MI communication remains efficient in stationary sensor networks and in mobile sensor networks, use of them is a challenging task and needs complicated mechanisms for reliable communication. For the mobile sensor modules, EM waves show better performances due to miniaturization of commercial transceivers. Researchers employ EM waves with supplementary mechanisms to facilitate reliable communication [35].

\section{B. Technical Gap}

Technical gaps in this field can be summarized as:

- A reliable wireless communication link is needed in harsh environment of propagation medium.

- There is not a reliable robotic system that can be used for water quality monitoring in distribution systems with large pipe diameters.

- In distribution systems, the range of pipe size change is high compared to other type of networks. To the best of our knowledge, there is no robotic system that can adapt to this rage of change.

- Operation environment in these applications are high pressure pipelines in which high speed flow is present. Hence, it requires a high demand for a robust control for motion control during operation.

- The mobility, control, wireless communication modules of mobile sensor modules need to be synchronized to achieve smart navigation during inspection. Smart navigation makes the robot negotiate special configurations, inclinations, and uncertainties in the network.

- Long and complicated pipeline network requires inspection methods to cover sufficiently large regions of pipelines. Hence, power supply is of paramount importance in inspection purposes and the tethered robots are no longer feasible to use.

- Pipelines comprise different configurations like straight paths, bends, Wyes, Tees. The in-pipe robots need a mechanism and motion control algorithm that facilitate motion in these configurations.

\section{Our Contribution}

Our contribution in this work can be listed in following: - We propose a modular in-pipe robot design so-called "SmartCrawler" that is dexterous and agile. It can adapt to wide range of size change in distribution systems compared to its counterparts. The underactuated design of the robot along with wheeled architecture and the flexible mechanism facilitate high manoeuvrability in the extremely disturbing environment of pipelines with fast motion. 
- We design a two-phase motion control algorithm for the robot that:

In phase 1: stabilizes the robot inside the pipeline in the straight paths while it tracks the desired velocity.

In phase 2: enables the robot to change its direction to the desired direction with reliable motion. We evaluate the performance of the proposed algorithm with simulation and experimental results.

- We propose a low frequency wireless communication setup that is bidirectional and facilitates sensors' data transmission from the robot to the base station and from the base station to the robot.

- We provide an empirical assessment of the proposed wireless communication setup performance with experimental results.

- We design an embedded system that synchronizes the wireless communication system with control and actuation units. The design follows the safety consideration of the operation environment of potable water.

\section{Paper Organization}

The paper is organized as follows: In section Section II the robot design, characterization, modelling, and fabrication is explained. In section Section III a two-phase motion controller is designed that controls the motion of the robot in straight paths and non-straight paths like bends and T-junctions. We also evaluate the performance of the proposed motion control algorithm in section Section III. In section Section IV] the wireless communication system is designed and its performance is evaluated with experiments. In section Section V we design the electronic embedded system for the robot. The paper is concluded and we present the steps for the future steps in section Section VI

\section{SMARTCRAWLER DESIGN, MODELLING, CHARACTERIZATION, AND FABRICATION}

\section{A. Design}

The robot is composed of a central processor and three actuation arm modules (see Fig. 1). We explain each module in detail as follow:

1) Central Processor Unit: The central processor, shown in Fig. 2] is composed of two parts. In one part, (part [c]), sensing elements are embedded. For the sensors that need water samples for measurement, a piezoelectric-based micropump system [45] provides samples by circulating water between two holes shown in [k]. This part of the central processor is socalled the sensing part. The other part of the central processor that is shown with [j], is the so-called the control part. In the control part, a printed circuit board (PCB) is located. In the $\mathrm{PCB}$, the modules that take sensor measurements controls the operation of actuators and establishes wireless communication between the robot and the base station is designed and located. The central processor needs to be sealed to protect the internal components. To this aim, we propose a seal mechanism with a standard off-the-shelf O-ring. In the seal mechanism, the O-ring is located between the sensing part and control part in which the sensing and control parts are then fastened with four pairs of screws and nuts. The screw-nut pairs, push the Oring and hence, seal the central processor. Elements of the seal mechanism are shown in [d], [e], and [f]. The arms shown in Fig. 1 are connected to the central processor with ball bearings. ([a] and [b] in Fig. 2). For each arm, two ball bearings are considered that are connected to both sides of the arm and facilitate rotation of arms concerning the central processor. Some supports are designed on the sensing and control parts to bound the range of rotation of the arms. The power source which is a compact battery is located in a case as shown in [h]. The battery power is transmitted to the central processor via wire through a sealed hole shown in [i]. We will provide detailed information about the embedded system in the central processor and various components that control the operation of the robot later in this paper.

2) Arm Module: Pipelines are composed of pipes of different sizes. The diameter of pipes changes during the operation of the robot. Hence, the robot's outer diameter needs to be adaptable to pipe size. To this aim, arm modules are designed in which three passive springs make the outer diameter of the robot adaptable to the pipe size (see Fig. 1). To address this requirement in our design, three passive springs are located between the arms and central processor (see Fig. 3). The anchor for connecting the end of each spring to the central processor are shown in $[\mathrm{g}]$ and on the arm. Each spring is connected to the central processor and the arm with a pair of dowel pins (see Fig. 3). The arms rotate about the central processor without friction, using ball bearings. Later in this paper, we provide more information about the spring mechanism characterization.

3) Actuator Module: At the end of each arm, an actuator module is located. The actuator modules in this robot are electrical motors equipped with gearhead and encoders to provide odometry of the robot and connected to wheels. The gear motors are located on the arms, fixed with designed motor covers, and directly connected to the wheels. The wheels are also connected and rotate about the arms with ball bearings. Hence, their motion is friction-free. The robot needs to stabilize itself during operation. Otherwise, it fails and stocks during operation. Retrieving a stuck robot from pipe is a costly and cumbersome task. This is much more sensitive when the network is in service as the region where the robot is stuck needs to shut down to retrieve the stuck robot. This requires the robot to be fully automotive. To this aim, the wheels in the robot should have pure rolling motion on pipe surface that means, sufficient friction is required between wheels and the pipe motion. In our design, in order to increase the friction between the wheels and pipe surface, we covered wheels with rubber. In the actuator module, the gear motor rotates the wheel and the wheel which is in contact with the pipe surface produces traction force for the robot and moves the robot forward inside the pipe. The CAD design of one actuator module, its components, and the way it is connected to the arm are shown in Fig. 4. We have three actuator modules in the robot that are powered and controlled by the central processor. 


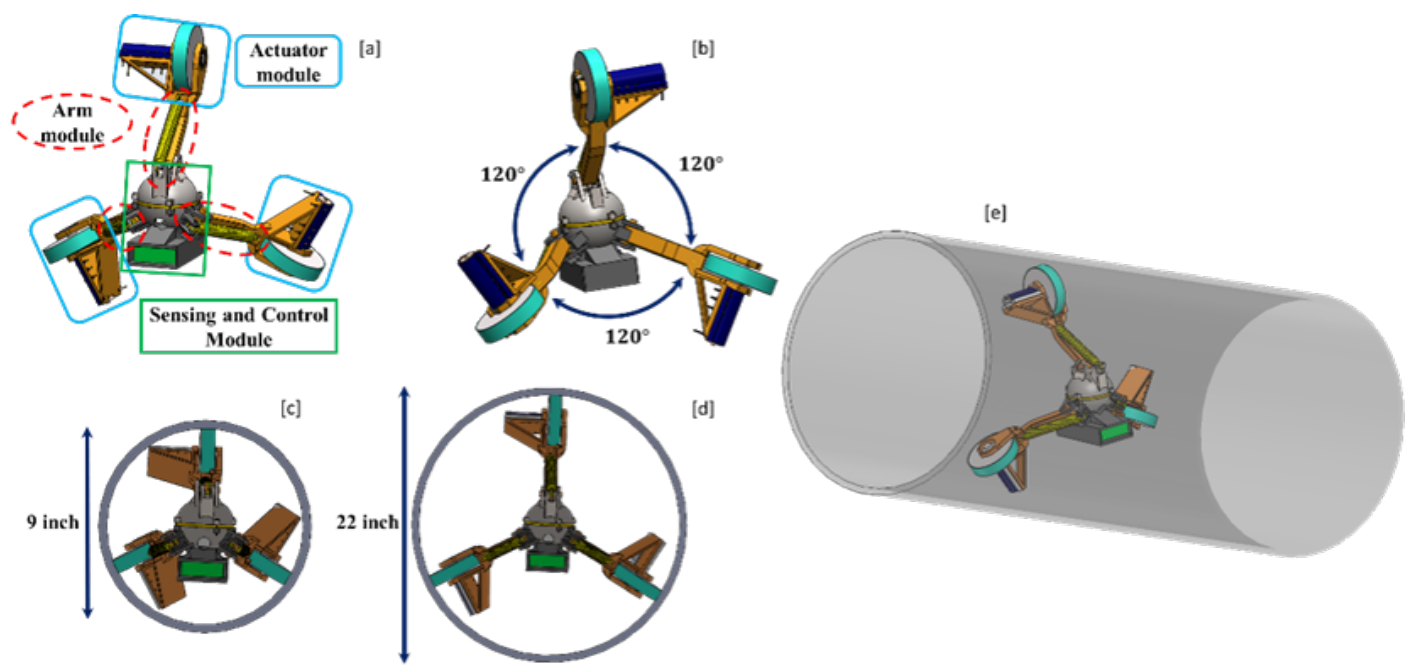

Fig. 1: In-pipe robot. a) CAD Design of the robot and its components. b) CAD design of the arms modules and their connection to the central processor. c) Robot with its minimum diameter and maximum diameter. e) The robot in pipe.

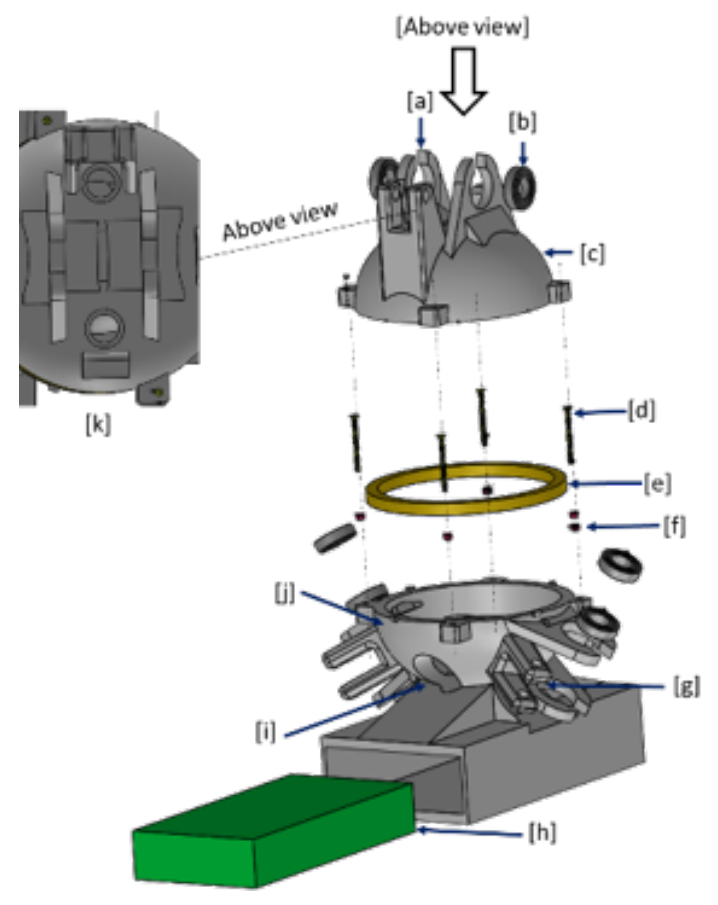

Fig. 2: Central processor. a) CAD design. [a] Arm basement. [b] Ball bearing. [c] Sensing hemisphere. [d] Screw. [e] Offthe-shelf O-ring. [f] Nut. [g] Spring anchor. [h] Battery. [i] Hole to connect inside and outside of the sphere electrically. [j] Control hemisphere. [k] Water inlet and outlet. b) Central processor prototype.

\section{B. Modelling}

The free body diagram of the robot in pipe is shown in Fig. 5. We present the degree of freedom (DOF) of the robot with linear velocity, $x$, rotation around $\mathrm{y}$-axis, $\phi$, and rotation around z-axis, $\psi$. The equations of motion of the robot are presented as follows:

$$
\frac{\tau_{1}}{R}+\frac{\tau_{2}}{R}+\frac{\tau_{3}}{R}+\frac{1}{2} \rho C_{d} A\left(\dot{x}-V_{f}\right)^{2}=m \ddot{x}
$$

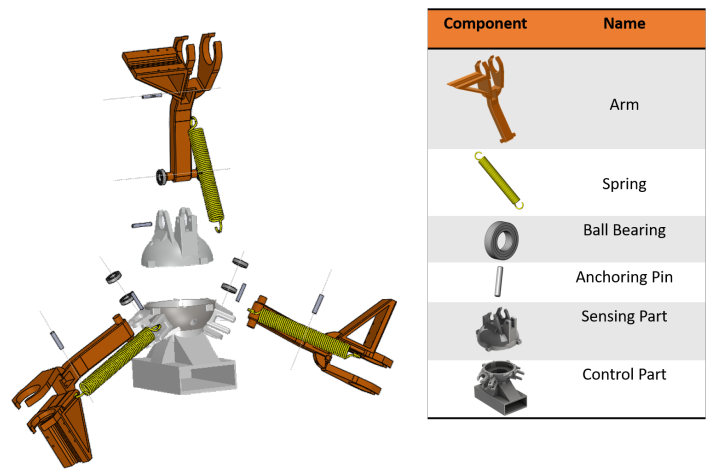

Fig. 3: Arm module architecture and the components.

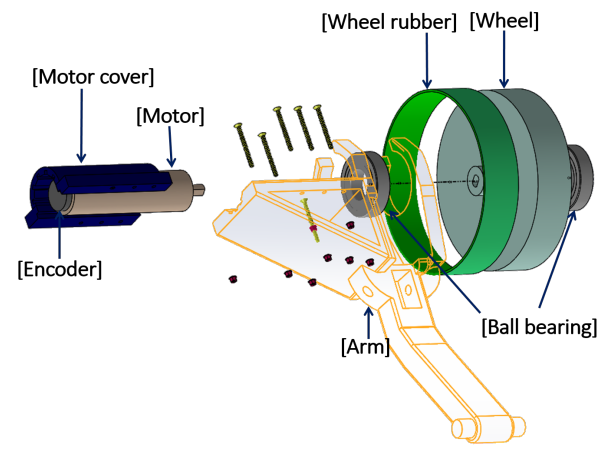

Fig. 4: CAD Design of the actuator module.

$$
\begin{gathered}
\frac{1}{2} \frac{\tau_{3}}{R} L_{a} \cos \left(\theta_{3}-\phi\right)-\frac{1}{2} \frac{\tau_{2}}{R} L_{a} \cos \left(\theta_{2}+\phi\right)=I_{y y} \ddot{\phi} \\
\frac{\sqrt{3}}{2} \frac{\tau_{3}}{R} L_{a} \cos \left(\theta_{3}-\phi\right)(1+\sin \psi) \\
+\frac{\sqrt{3}}{2} \frac{\tau_{2}}{R} L_{a} \cos \left(\theta_{+}+\phi\right)(1+\sin \psi) \\
-\frac{\tau_{1}}{R} L_{a} \cos \left(\theta_{1}+\right)=I_{z z} \ddot{\psi}
\end{gathered}
$$

$\tau_{i}$ and $R$ are the gear-motors torque and wheel radius, respectively. Full representation of the system's dynamical 

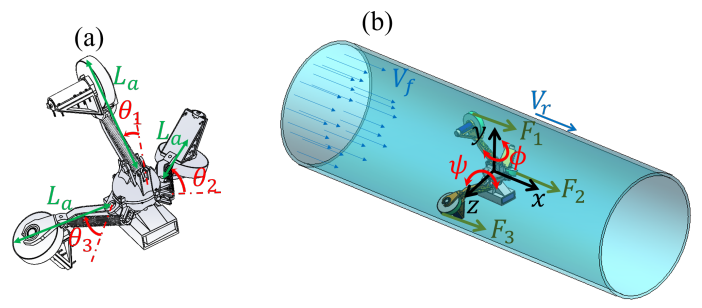

Fig. 5: (a) The robot geometry. (b)Free body diagram representation of the SmartCrawler in pipe.

TABLE I: CFD SIMULATION PARAMETERS

\begin{tabular}{|cc|}
\hline Parameter [unit] & Description \\
\hline \hline Fluid type & Water \\
Fluid pressure $[\mathrm{kPa}]$ & 413 \\
Fluid density $\left[\mathrm{kg} / \mathrm{m}^{3}\right]$ & 1000 \\
Fluid velocity $[\mathrm{m} / \mathrm{s}]$ & 0.6 \\
Robot velocity $[\mathrm{m} / \mathrm{s}]$ & 0.5 \\
The relative direction of the robot and flow & Opposite \\
\hline
\end{tabular}

equations is presented in our previous work [36].

\section{Characterization}

The robot operates in WDS while the system remains in use and the pressurized operation environment affects the robot parameters. Besides, the robot parameters need to be modeled and characterized. The drag force [36] is the dominant force against the robot during operation depends on the relative velocity of the robot and the flow and computed as:

$$
f_{d}=\frac{1}{2} m C_{D} A V_{r e l}^{2}
$$

where $m$ is the robot mass, $C_{D}$, the drag coefficient of the robot, , water flow density, and A is cross-sectional area is the robot facing the flow. $V_{\text {rel }}$ is the relative velocity of the robot and flow:

$$
V_{\text {rel }}=V_{r}-V_{f}
$$

where $V_{r}$ and $V_{f}$ are the robot and the flow velocities, respectively (Fig. 6). Drag force is not constant for the robot and changes with $V_{r e l}$ and the outer diameter of the robot. To calculate the maximum possible drag force, we simulated the operating environment with CFD work. In the CFD work, an extreme condition is simulated where the robot is in the extended position. The robot and the flow move against each other in the simulation. The objective of the CFD work is to first evaluate the robot interference with the flow during operation and then, calculate the maximum drag force. In Fig. 6, the simulation environment is shown. The colors in the figure show the relative flow velocity in the pipe $\left(V_{\text {rel }}\right)$ and in the vicinity of the robot. Water pressure is $413 \mathrm{kPa}$ [37], and the flow velocity is $0.60 \mathrm{~m} / \mathrm{s}$. Besides, the robot velocity is $0.5 \mathrm{~m} / \mathrm{s}$. The largest relative velocity, as shown in blue, is $1.2 \mathrm{~m} / \mathrm{s}$, which is the relative velocity of the flow to the robot. In table I, the simulation parameters are shown. In this simulation, the maximum drag force was computed to be around $18 \mathrm{~N}$.

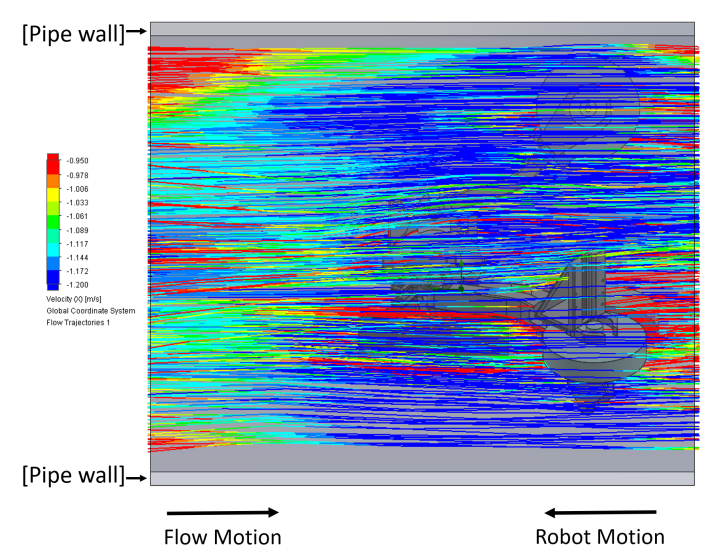

Fig. 6: CFD work In SolidWorks for maximum drag force computation.

1) Spring Stiffness: For reliable motion in pipe, the robot needs sufficient friction force between its wheels and the pipe wall. The friction force is provided by spring force in the robot. We characterized the spring force based the CFD work (explained earlier) in our previous work [38].

2) Power Consideration: We also did a power profiling for the electronic components of the SmartCrawler and provided a recursive approach in our previous work that suggests the minimum battery capacity and the operation duration in the extreme condition (CFD work) [38] .

3) Gear-motor: We also selected the customized gear-motor based on the CFD work in our previous work [39]. The gearmotor in the SmartCrawler provides sufficient torque to tackle the drag force and move the robot in pipelines.

\section{Fabrication}

In this section, we present the fabrication process of the robot. The wheels, arms, sensing and control parts, the motor cover, and the wheel rubber are 3D printed with acrylonitrile butadiene styrene (ABS) material. The pins that connect the springs to the arms and the central processor in the arm modules are commercial off-the-shelf 416 stainless steel dowel with a 0.18 -inch diameter and 0.875 -inch length. Since the robot operation environment is in potable water in pipelines, it should not be a source of toxic materials and also the robot components should not interact chemically (e.g. oxidize) with the water during fabrication. We address the health issue considerations in the design as presented in follows:

- The ball bearings of the robot are sealed.

- On the motor covers in the actuator modules, some slots are designed and filled with mouldable glues to seal and fix gear motors on the arms. In the glue, non-toxic materials are used. - The battery is placed on a designed case on the control hemisphere and sealed with moldable glue.

- The electronic parts in the central processor are sealed.

- The springs, anchoring pins, screws, and nuts are stainless steel in which would not oxidize in water.

- Since water pressure is high in pipelines [48], the body of the robot (especially the central processor) is designed to bear a high-pressure environment. 
TABLE II: SMARTCRAWLER SPECIFICATIONS

\begin{tabular}{|cc|}
\hline Parameter [unit] & Description \\
\hline \hline Size change range capability [inch] & $9-22$ \\
Length [inch] & $7.5-9$ \\
Maximum linear speed [m/s] & 1.56 \\
Weight $[\mathrm{kg}]$ & 2.23 \\
\hline
\end{tabular}

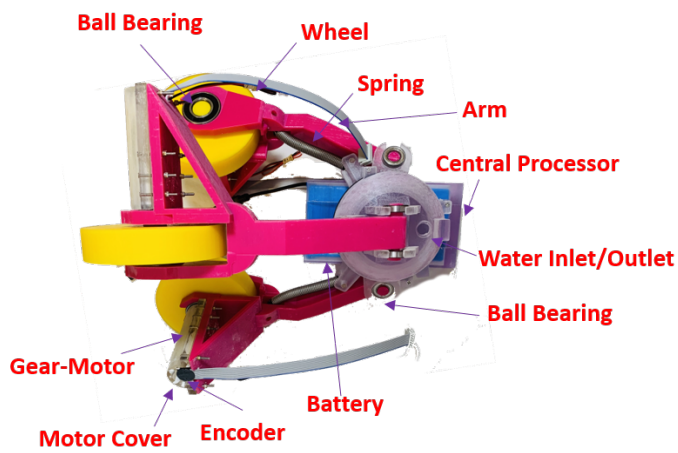

Fig. 7: The prototype of the system.

The robot general parameters are listed in Table II The robot prototype is shown in Fig. 7 The modular design of the robot facilitates easy disassembling and maintenance.

\section{TWO-PHASE CONTROL DESIGN, SIMULATION, EXPERIMENT, AND DISCUSSION}

There is high pressure in the robot's operation environment. Also, since the robot is designed to operate in pipelines while the network remains in service, water flow is present. We explained that if the robot gets stuck in the pipeline during operation, a costly and difficult task needs to be done to retrieve it from the system. Hence, the robot needs to be fully automotive. So far, we simulated an extreme operating condition with CFD work, and computed maximum drag force applying on the robot. Based on the extreme condition, the spring mechanism was characterized in our previous work to ensure sufficient contact force for the robot. Also, power profiling is done for the robot based on the operation condition and a method is presented to calculate the minimum battery capacity to power the robot's components and operation duration in extreme conditions.

In this section, we propose a two-phase motion controller algorithm. In phase 1, the controller stabilizes the robot in the pipe during operation. The "stabilizer-velocity tracker" controller enables the robot to move smoothly in straight paths. However, the robot operation is not always a straight path. Pipelines comprise different configurations like bends and Tjunctions. We add another phase to the controller algorithm (i.e. phase 2) that enables the robot to pass through T-junctions and bends, which are common non-straight configurations in pipelines. First, we design the stabilizer-velocity tracker controller in Section III-A and evaluate its performance with ADMAS MATLAB co-simulation and also experiments. Then, in Section III-B, we design the phase 2 and add it to the stabilizer-velocity tracker controller to form the two-phase controller. We evaluate the performance of the phase 2 with ADAMS MATLAB co-simulation.

\section{A. Phase 1: Stabilizer-velocity Tracker}

A dynamical system can be represented as:

$$
\begin{array}{r}
x(t)=A x(t)+B u(t) \\
y(t)=C x(t)
\end{array}
$$

In Eq. (6), $x(t)$ is the system state vector and represents the physical parameters of the system. $A$ is the system matrix, $B$ is the input matrix, $C$ is the output matrix, and $y(t)$ is the output matrix. In the LQR method, a cost function is defined which is the combination of state functions and input control efforts, and the controller tries to minimize it. The cost function is [40]:

$$
J(K)=\frac{1}{2} \int_{0}^{\infty}\left[x(t)^{T} Q x(t)+u(t)^{T} R u(t)\right] d t
$$

where $Q$ matrix weights the state vector and penalizes the cost function in case a deviation for the states is observed. $R$ matrix penalizes the cost function to have minimum control effort, $u(t)$. The control input in the LQR method is computed as:

$$
u(t)=-K x(t)
$$

where $K$ is the gain matrix and is calculated as:

$$
K=R^{-1} B^{T} P
$$

In Eq. (9), $P$ matrix is computed based on Riccati equation:

$$
-P A-A^{T} P-Q+P B R^{-1} B^{T} P=0
$$

Klemm et al [41] have shown LQR-assisted controller has high robustness and reliability for a two-wheeled mobile robot.

We consider $x(t)=\left[\begin{array}{llll}\phi & \dot{\phi} & \psi & \dot{\psi}\end{array}\right]$ as stabilizing states that need to be kept at zero value during operation. The robot is stabilized with $x(t)$. The system dynamic equations are nonlinear. To derive the state-space representation for the system to compute the gain matrix, $K$ for LQR stabilizer, we linearize the system equations that can be generally represented as $x(t)=f(x(t), u(t))$ around an equilibrium point, $x_{e}$. Hence:

$$
\dot{x}(t)=f\left(x_{e}+\delta x, u_{e}+\delta u\right)
$$

where $u_{e}$ is the controller input at the equilibrium point. We can write based on Taylor expansion [42]:

$$
\delta \dot{x}=\frac{\partial f}{\partial x} \delta x+\frac{\partial f}{\partial u} \delta u+H O T(x)+H O T(u)
$$

$H O T(x)+H O T(u)$ are higher-order terms that are neglected. The system matrices are written as:

$$
\delta \dot{x}=A \delta x+B \delta u
$$

Comparing Eq. (13) with Eq. [12], the system matrices are derived as:

$$
\begin{aligned}
A & =\frac{\partial f}{\partial x} \\
B & =\frac{\partial f}{\partial u}
\end{aligned}
$$

The LQR gain matrix is computed with system matrices in Eq. (14). So far, the stabilizer controller is designed based on the optimal LQR controller to enable the robot to negotiate disturbances applied on the robot during operation. It also 


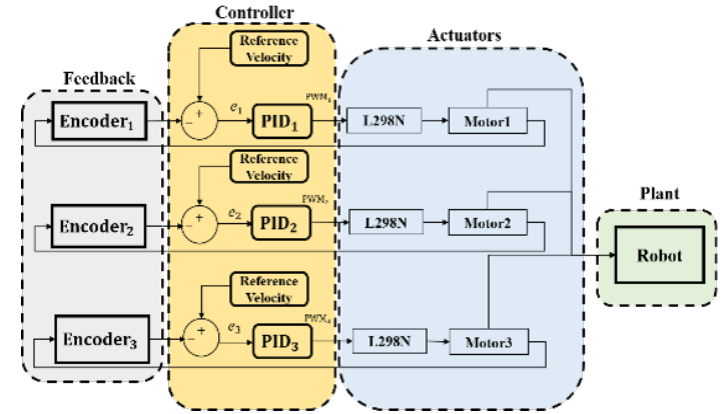

Fig. 8: The Velocity controller algorithm for the robot.

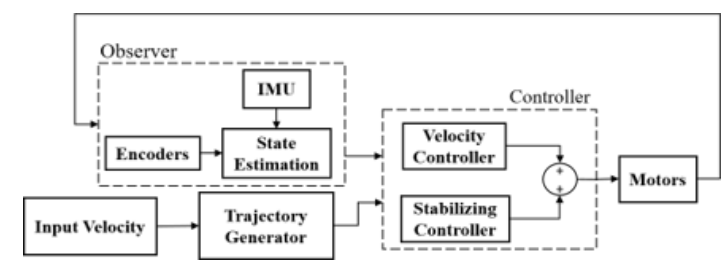

Fig. 9: The controller to stabilize the robot and track the desired velocity in straight paths.

keeps the central processor at the center of the pipe to lower drag force [38].

The robot has controlled motion in the pipeline which is independent of flow. To track a defined velocity, we design a PID-based controller. The encoders in gear motors provide odometry of the robot. The robot's wheel angular velocity are equal in the straight path, hence the relation between the linear velocity of the robot and the encoder output is calculated as:

$$
v=\frac{2 \pi R}{N T_{c}}
$$

where $N, R$, and $T_{c}$ are the number of pulses per wheel turn that encoders generate, wheel radius, and the time between two consecutive pulses, respectively. In the velocity controller, the desired velocity is translated to the desired angular velocity based on Eq. 15. Then, three PID regulators keep the all wheels' angular velocity at the desired angular velocity (see Fig. 8).

The LQR stabilizer and velocity controllers are combined and shown in Fig. 9. The observer in Fig. 9, provides robot odometry and orientation. The input velocity is the desired linear velocity. The trajectory generator in the proposed controller computes the desired angular velocity for the wheels with input velocity.

1) Controller Performance Evaluation ADAMS-MATLAB CoSimulation: To evaluate the controller performance, we modeled the robot in ADAMS, dynamic simulation software, and co-simulated it with the designed controller in MATLAB 2020. In this way, the controller is implemented in the MATLAB Simulink toolbox and connected to the ADMAS in real-time during the simulation. The stabilizing states $(\phi$ and $\psi)$ and the robot linear velocity is computed in ADAMS and reported to the controller in MATLAB Simulink in real-time. The controller computes the input control signal, which is motor torques and sends it to ADAMS to apply on the wheels. In Fig. 10 the simulation results are shown. The coordinate system in the simulations is the same as shown in Fig. 6. It should be mentioned that in this simulation, the wheels are not in contact with the pipe wall at the beginning of the simulation. Hence, there is a fluctuation in the linear velocity of the robot (Fig. 10a) and angular velocities of the robot (Fig. 10b) for the first $0.1 \mathrm{sec}$ of simulation. After the wheels contacted the pipe wall, the fluctuations converged to zero. In Fig. 10a, the legends define the wheel's angular velocities in revolutions per second (RPM). The robot moves in the pipe axis with desired velocities; $0.12 \mathrm{~m} / \mathrm{s}, 0.17 \mathrm{~m} / \mathrm{s}$, and 0.35 $\mathrm{m} / \mathrm{s}$ with stabilized reliable motion (i.e. angular velocities are zero). In Fig. 10b, the angular velocities of the robot along each axis $(x, y$, and $z)$ are shown for the case the desired robot velocity is $0.35 \mathrm{~m} / \mathrm{s}$. In Fig. 16b, the angular velocity of the robot on each axis ranges between $-4 \% \mathrm{sec}$ to $+3 \% \mathrm{sec}$. We also considered external disturbances in our simulations to evaluate the robustness of the controller in presence of flow. The simulation results show the feasibility of the controller for the straight path as the robot can track a specific velocity in the pipe with balanced orientation. The simulation results show that the proposed control strategy is capable of maintaining the desired velocity for the robot with minimal rotation in the pipe [38].

2) Controller Performance Evaluation with Experimental Results: A testbed is developed to evaluate the performance of the stabilizer-velocity controller (see Fig. 11a) [38]. An inertial measurement unit (IMU) that includes an accelerometer and gyroscope (BMI160 breakout from Bosch Sensortec.) is located at the center of the robot on the central processor. The IMU sends linear acceleration and gyro data of the robot to the microcontroller unit (MCU) via an inter-integrated circuit (I2C) protocol. The Arduino Mega2560 microcontroller board based on the ATmega2560 microcontroller is used as the MCU. Three ENX EASY 16 incremental encoders with six channels that work with Hall Effect and provide angular velocities of the motors are connected to the MCU. To control the velocity of the motors and also their direction, L298N, dual full-bridge motor driver are used. Each driver board controls two motors. As we have three motors, two L298N boards are used. We used a 15 A.h super lithium-ion battery with $12 \mathrm{~V}$ to power the actuation units (i.e. gear-motors). The pipe that the robot moves in is a 14-inch diameter SCH.40 PVC pipe. In Fig. 11a, the motion direction of the robot is shown in the experiment. Since the IMU outputs are noisy and have a DC baseline, it is not feasible to calculate the rotational angles, directly from the IMU (i.e. by integration). Hence, we computed the rotational angles with Mahony complementary filter [43] which is an optimal sensor fusion to calculate roll, pitch, and yaw angles with the IMU outputs. The robot's linear velocity is computed by the encoders' output with Eq. (15). The control algorithm is programmed in the Arduino Integrated Development Environment (IDE) and connected to MATLAB2020 with MATLAB support package for Arduino hardware. We monitored the velocity of the robot, and Mahony filter angles $(\phi$ and $\psi)$ in MATLAB2020 in real-time. We evaluated the performance of the developed control algorithm 


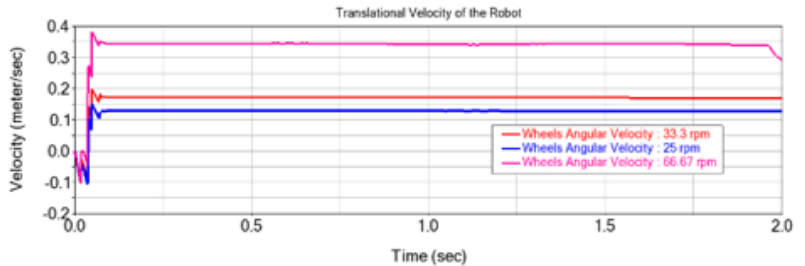

(a)

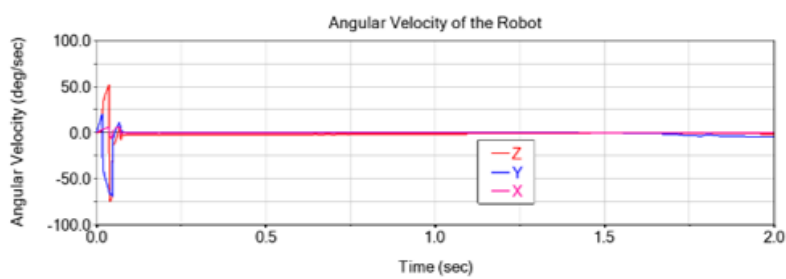

(b)

Fig. 10: The robot functionality simulated in ADAMS in the 1.5-meter straight pipe based on the EVA control strategy. (a) The velocity of the robot for different desired transnational velocities. (b) The robot angular velocity around its axes. The fluctuations at the beginning of the simulation are due to the fact the wheels are not in contact with the pipe at the start of the simulation.

with different initial values for $\phi$ and $\psi$ and desired linear velocities in four iterations (see Fig. 18). In Figs. 18a, 18b, and $18 \mathrm{c}$, the robot velocity and the robot-controller performance for and are shown, respectively. Each curve shows the results for each iteration. The robot is at rest (i.e. zero velocity) at the time each iteration starts. The stabilizing states at the time each iteration starts, $\phi_{0}$ and $\psi_{0}$, are non-zero [38].

In iteration 1 (The blue curve in Fig. 18), $\phi_{0}$ and $\psi_{0}$ are $-4^{\circ}$ and $-3^{\circ}$, respectively. Besides, they converge to zero and fluctuate around zero value with $\pm 2^{\circ}$ margin in around two seconds. The fluctuation margins are the same for other iterations as well. In this iteration, the desired linear velocity for the robot is $0.1 \mathrm{~m} / \mathrm{s}$. The robot reaches the desired velocity in around two seconds. In iteration 2 (The red line in Fig. 19), the initial value for $\phi_{0}$ and $\psi_{0}$ are $-14^{\circ}$ and $-11^{\circ}$, respectively; and the desired linear velocity for the robot is $0.2 \mathrm{~m} / \mathrm{s}$. The stabilizing states converge to zero in two seconds and the robot reaches $0.2 \mathrm{~m} / \mathrm{s}$ velocities in less than three seconds. In iteration 3 (The orange line in Fig. 19p, $\phi_{0}$ and $\psi_{0}$ are $-9^{\circ}$ and $+5^{\circ}$, respectively. The robot reaches to desired linear velocity (i.e. $0.3 \mathrm{~m} / \mathrm{s}$ ) in four seconds and stabilizes the stabilizing states in one second. The stabilization duration is again around one second in iteration 4 (The purple line in Fig. 19) and the time the robot reaches the desired linear velocity of $0.35 \mathrm{~m} / \mathrm{s}$ in five seconds. The experimental results prove that the developed stabilizer-velocity tracker controller can control the velocity of the under-actuated robot and stabilize it in the highly uncertain environment of pipelines. For example, over the course of time, the minerals in water cause sediments in pipes, which make the internal shape of the pipe non-circular, and there is no prior information about them. Another example is phalanges in pipes that are used to create junctions and bends. The phalanges make a rapid and sudden change in pipe shape that requires the robot's control algorithm to stabilize the robot against the impact these sudden shape changes impose on the robot. The developed controller negotiates these uncertainties and disturbances and stabilizes the robot against its weight.

\section{B. Phase 2: Controller Algorithm for Non-Straight Paths (i.e. $90^{\circ}$-bends and T-junctions)}

Fig. 14 shows the two-phase controller that controls the motion of the robot in straight and non-straight paths. The robot switches between two phases of the control dependent on the path. We focus on the phase 2 in this part. The controller in this phase, first calculates the rotation angle around y-axis (i.e. $\phi_{d}$ ) that is either $+90^{\circ}$ or $-90^{\circ}$ considering desired direction. Based on $\phi_{d}$, the controller sets the desired velocity for each wheel based on a method so-called "Variable Velocity Allocation (VVA)". In VVA, the wheels track different desired velocities based on $\phi_{d}$ ("Trajectory Generator" block in Fig. 147. The trajectory generator sets the desired values for wheels based on Fig. 12

The trajectory generator sets the desired values for the wheels and the PID-based control block follows the desired velocities. An "Error check submodule" block (Fig. 14, continuously checks the amount of rotation and based on this amount, decides to either continue on the current control phase or switch to stabilizer-velocity tracker controller (see Fig. 13). When the rotation of the robot is completed, the controller switches to the stabilizer-velocity tracker controller.

1) Controller Performance Evaluation at $90^{\circ}$ Bend: In this part, the performance of the controller in phase 2 is evaluated at $90^{\circ}$ bend with simulation. The robot in a pipe with a 12-inch diameter is shown in Fig. 16a. The front path is a $90^{\circ}$ bend and the robot needs to rotate $90^{\circ}$ clockwise around the y-axis (i.e. $\phi_{d}=-90^{\circ}$ ) to pass through the bend. The coordinate system is considered on the robot and moves with it. The sequence of motion during simulation is shown in Fig. 16b with numbers from one to six. In photo labelled number one in Fig. 16a, the robot's wheels are marked with three colored circles. Based on the controller in phase 2 , the wheels with green circle mark has maximum angular velocity (i.e. $60 \mathrm{RPM}$ ), the wheel with red circle mark has minimum angular velocity (i.e. 30 RPM), and the wheel with black circle mark has the velocity of the average of green and red circles marked wheels' angular velocity (i.e. 45 RPM). In the sequences of motion, the dotted arrows show the length each wheel has travelled in the bend in each sequence. The green wheel travels longer distances compared to the black and red wheels.

In Fig. $16 \mathrm{~b}$, the robot's angular velocities around three axes 


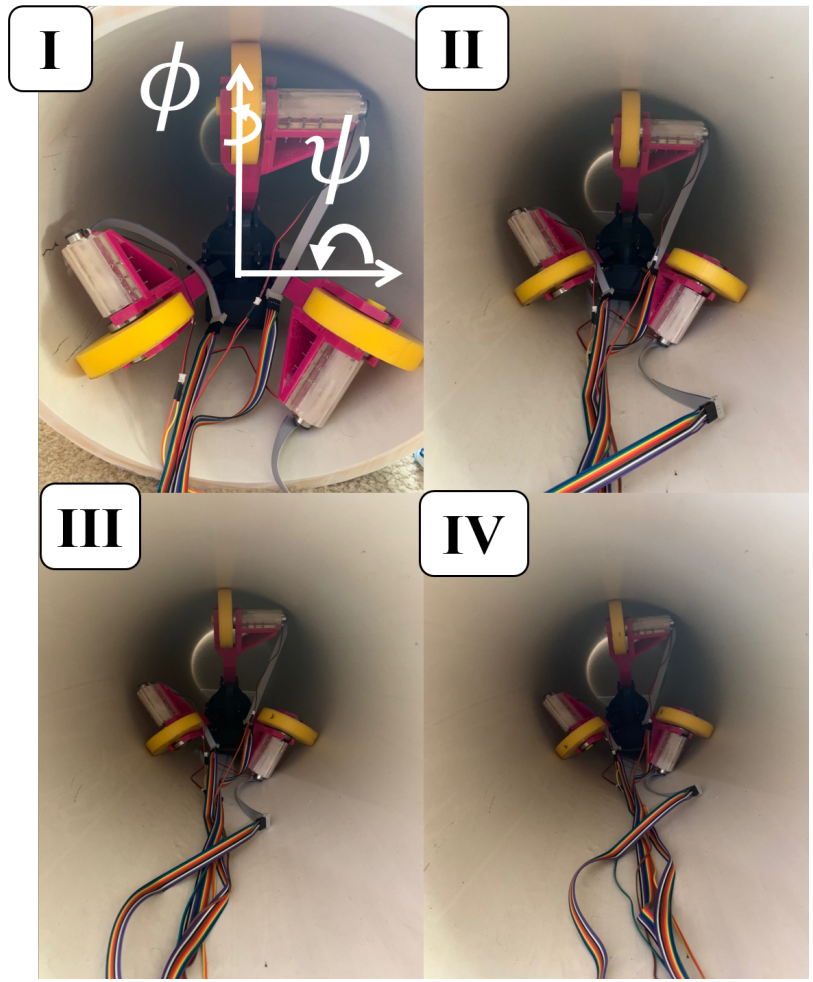

(a)

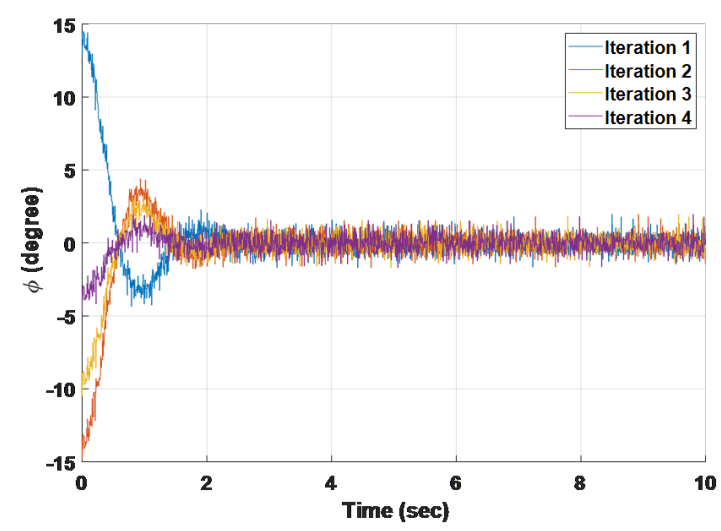

(c)

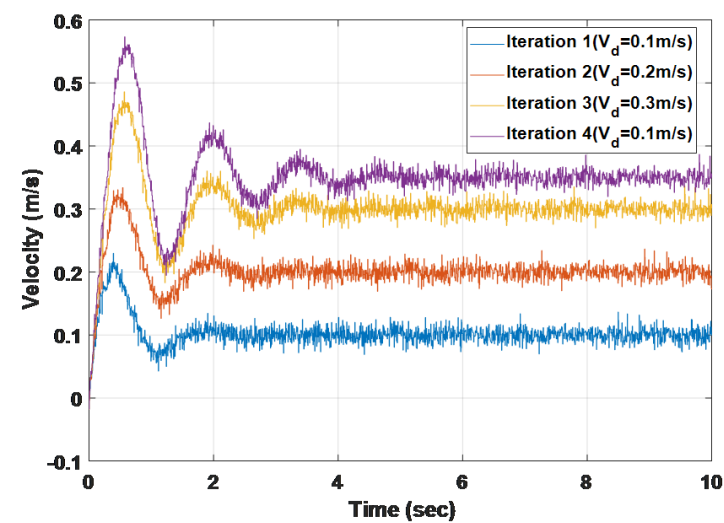

(b)

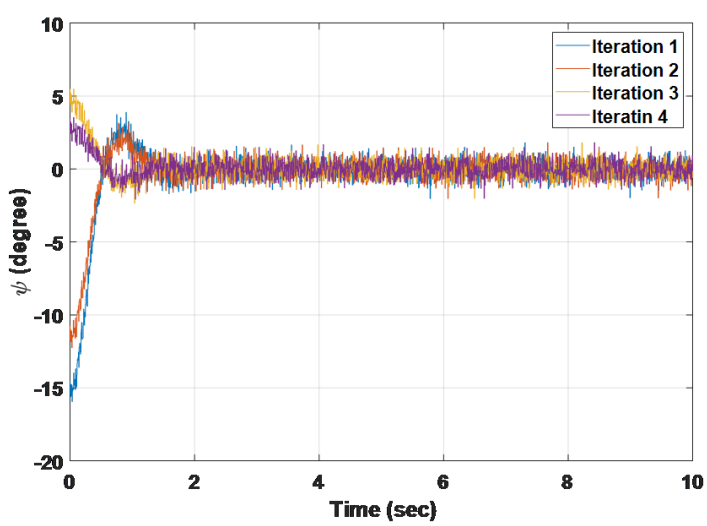

(d)

Fig. 11: (a) Sequences of motion of the robot with the stabilizer-velocity tracker controller. (b) Linear velocity (m/s). b) $\phi$ (degree). c) $\psi$ (degree).

(x-axis, $\mathrm{y}$ - axis, and z-axis) are shown. The blue curve is the angular velocity around the y-axis. The robot's angular velocity around the $y$-axis ranges between $-10 \%$ and $-25 \%$ s. The pink-colored curve shows the angular velocity of the robot around the $\mathrm{x}$-axis. The angular velocity around the $\mathrm{x}$-axis ranges between $+1 \%$ to $-5 \%$ during motion. The red curve in Fig. $16 \mathrm{~b}$ shows the robot's angular velocity around the z-axis that ranges $\pm 1 \%$ s during motion. The angular velocities around the axes show that the robot rotates $-90^{\circ}$ around the $y$-axis, $-8^{\circ}$ around the $\mathrm{x}$-axis, and $\approx 0^{\circ}$ around the $\mathrm{z}$-axis.

The rotation angles around each axis during this simulation show the robot along with the developed controller can pass through bends smoothly. One of the issues that in-pipe robots face during passing through bends is that they get stuck during rotation as a result of their dimension. Chatzigeorgiou et al. developed a design criterion in [48] which defines the maximum allowable length to height ratio (see Fig. 15) for inpipe robots in bent pipes. Our robot meets this design criterion since the arms are highly flexible and also, the height of the robot decreases as its length increases.

2) Controller Performance Evaluation in T-junctions: In this section, we evaluate the performance of the phase 2 controller in T-junctions. The robot in a 12 -inch diameter pipe with a Tjunction path ahead is shown in Fig. 17a In photo number one, the wheels are marked with three colored circles. The robot 


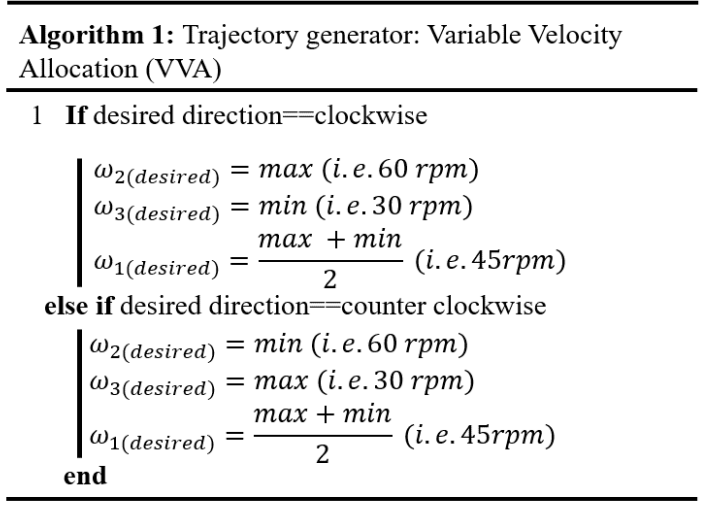

Fig. 12: Variable Velocity Algorithm (VVA).

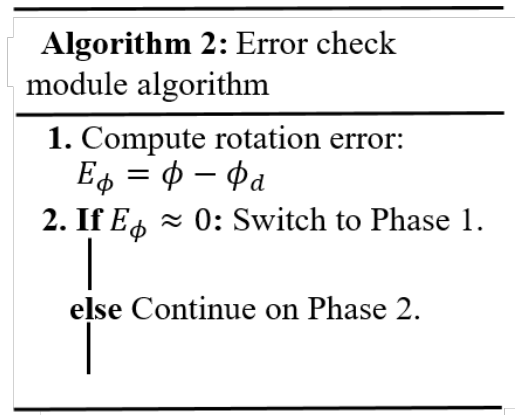

Fig. 13: Error submodule algorithm.

needs to rotate $90^{\circ}$ counterclockwise around the y-axis (i.e. $\phi_{d}=+90^{\circ}$ ). The green-circle-marked wheel (green wheel for brevity) has maximum velocity.

The red-circle-marked wheel (red wheel) has minimum velocity and the black-circle-marked wheel (black wheel) has a velocity which is the average velocities of green and red wheels. The coordinate system is on the robot and moves with it during motion. The robot starts moving and the green wheel loses its contact with Pipe (I) and due to pretension in springs, contact with the Pipe (II) wall. The trajectory of the green wheel is shown with a dashed green arrow. The red and black wheels smoothly enter Pipe (II). The robot's angular velocities around the $\mathrm{x}$-axis, $\mathrm{y}$-axis, and $\mathrm{z}$-axis are shown in Fig. $17 \mathrm{~b}$ along with the robot's linear speed. In Fig. $17 \mathrm{~b}$, the right vertical axis shows the linear speed in $\mathrm{m} / \mathrm{s}$ and the left vertical axis shows angular velocities in \%. The solid blue curve shows the robot's angular velocity around the y-axis. The velocity is between $+25^{\circ}$ /s and $+75^{\circ}$ /s most of the time. However, in a short period, the velocity reaches $+200 \%$ and $-50 \%$. The solid red curve shows the robot's angular velocity around the $\mathrm{x}$-axis. It has values range between $-37 \%$ and $+25 \%$ s during motion and fluctuate between these two values. The solid pink curve shows the robot's angular velocity around the $\mathrm{z}$-axis. The angular velocity around the $\mathrm{z}$-axis oscillates between $-50^{\circ} \% \mathrm{~s}$ to $+100^{\circ} / \mathrm{s}$ and most of the time is positive. At the end of the rotation, the rotation around the z-axis is not near zero as can be seen in Fig. 17a, photo number six. The dotted blue curve shows the robot's linear speed during rotation. The values for linear velocity start from zero and range between $0.15 \mathrm{~m} / \mathrm{s}$ and $0.25 \mathrm{~m} / \mathrm{s}$. However, the robot's speed is constant and it rotates smoothly in the motion. The simulation results show the proposed controller at phase 2 enables the robot to change its direction in the challenging environment of T-junctions as there is minimum available contact space for the wheels. The size-adaptable mechanism of the robot along with this control scenario makes rotation in the T-junction possible with a smooth motion. The pretension in the springs makes the wheels be in contact with pipe walls most of the time during rotation. Also, the duration at which the wheel loses its contact with the pipe wall is small enough that the robot will not collapse in the junction. As mentioned, there is a rotation around the z-axis at the end rotation. The controller switches to phase 1 when the error check submodule defined a complete rotation of the robot. Therefore, the undesired rotation around the z-axis is zeroed with the stabilizer-velocity tracker controller.

So far, we designed an in-pipe robot, characterized it to be fully automotive in pipelines, and designed a two-phase controller to enable it to move in different configurations of pipelines. In other words, the mechanical aspect of the robot is discussed. In Section IV] and Section [V] we focus on the electronic part of the SmartCrawler as follows:

1) Design a wireless communication setup for underground applications and evaluate its performance with experiments.

2) Design the printed circuit board (PCB) that is the "brain" of the robot and control all functions of the robot during operation.

\section{WIRELESS COMMUNICATION}

Wireless underground communication (WUC) is a challenging task. High path loss, dynamic communication channel, and space limitations for antenna embedding are challenges about the WUC. In this section, we propose a wireless communication system that works based on active low-frequency (RFID) technology. The sensor measurements can be transmitted wirelessly in the harsh environment of water where signal attenuation is high. The bi-directionality capability of our proposed wireless communication system enables it to be synchronized with the actuation and control units of the robot. We first present the RFID technology in summary and present our communication setup. The functionality of the proposed communication setup is evaluated with experimental results.

\section{A. Radio Frequency Identification (RFID)}

RFID is the technology where two transceivers communicate whenever they are close to the range of each other [44]. In RFID technology, seven layers of communication (open system interconnection Model (OSI)) [45] are abstracted [44] and there is no need to synchronize the connecting devices before data transmission starts. This feature of RFID systems makes them ideal candidates for the applications in which a device switches its communication during operation. In Fig. 18, the operation principle of a full-duplex (FDX) RFID where tag and reader can send and receive data are shown. RFID modules 


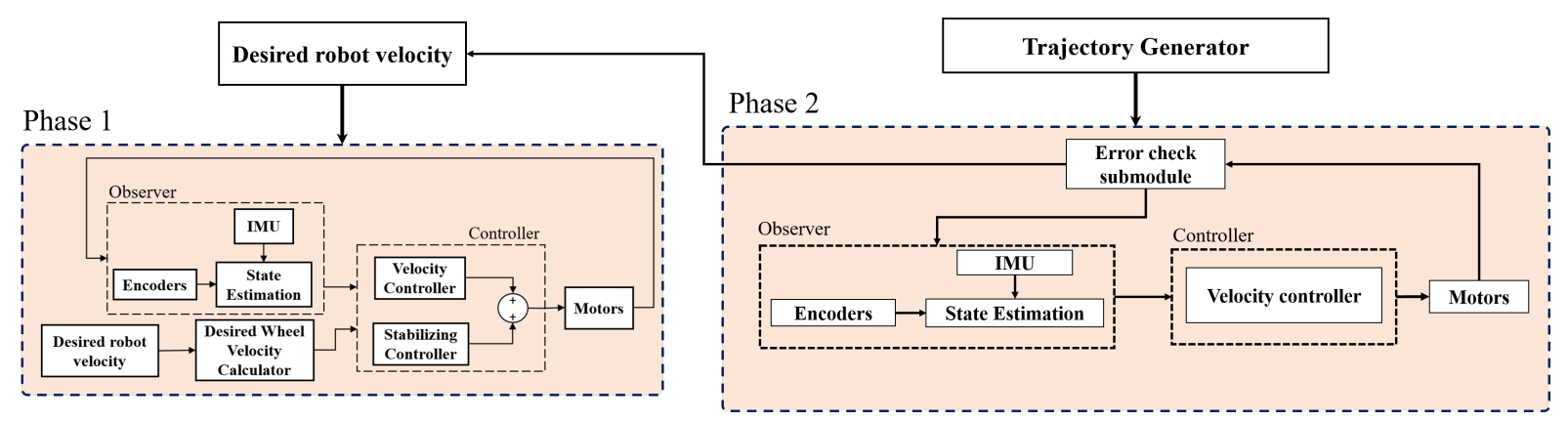

Fig. 14: The control strategy to control the robot.

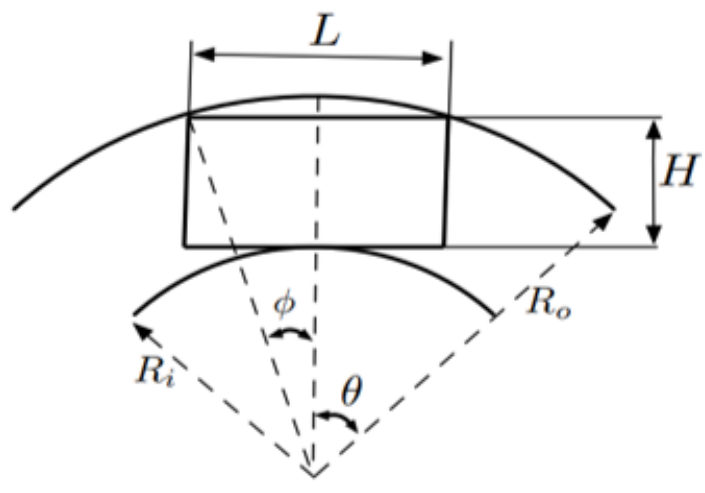

Fig. 15: The 2D sketch to define the maximum allowable length $(\mathrm{L})$ to height $(\mathrm{H})$ ratio for the robot in bent pipes. Ri and Ro are inner and outer diameters of the bent pipe. The photo is adopted from [48].

work in different carrier frequencies. Based on the operating frequency, they are categorized into low frequency (LF), high frequency (HF), and ultra-high frequency (UHF) modules [44]. This feature is also desirable in applications where there is high path loss in environments like underground applications and low-frequency signals are required for efficient realization. The tags can be passive or active. Passive tags lack energy sources and processing units. When the tag is within the read range of a reader, the energy is provided by the reader by magnetic or EM field and the tag data are transmitted. While active tags have their MCU and energy source. Also, the RFID technology can be in read, or read and write modes [44]. In other words, the tag and reader can both send and receive data.

\section{B. RFID Communication Setup in SmartCrawler Robot}

In this research, we implement a read and write RFID module where both sides of the wireless communication system can receive and send data. To this aim, we use Texas Instruments (TI) Inc $\odot$ technology, $\mathrm{CC} 1200$ as the physical layer. CC1200 is fully integrated low-power chip [56]. The variable sub-1 GHz carrier frequency, which CC1200 can work in, makes it an ideal option for our application. It works in three frequency bands; $164-190 \mathrm{MHz}, 410-475 \mathrm{MHz}$, and 820$950 \mathrm{MHz}$. In addition, it is possible to put the RFID chip in either transmit or receive mode with command strobes by the host MCU. The interrupt feature of the CC1200 helps to put in a low-power mode when there is no need for communication
TABLE III: RFID PHYSICAL LAYER PARAMETERS

\begin{tabular}{|cc|}
\hline Parameter [unit] & Description \\
\hline Transceiver IC & CC1200 \\
MCU & Arduino Mega2560 \\
Communication type with the MCU & SPI \\
Data FIFO [byte] & 125 in TX, 125 in RX \\
Maximum data rate [kbps] & 1250 \\
Carrier frequency [MHz] & 434 \\
\hline
\end{tabular}

and it helps saving power. $\mathrm{CC} 1200$ is connected to the MCU via a serial peripheral interface (SPI) connection. CC1220 has 128 bytes data first input first output (FIFO) buffer on both receiver and transmission sides, where facilitates up to 128 bytes storage on the IC on both transmit (TX) and receive (RX) sides. More details on this RFID IC can be found in [56]. To configure desired functions of the CC1200 IC (e.g. carrier frequency, transmit power, modulation format, symbol rate, etc.), register configuration is needed. Full register configuration of CC1200 is facilitated with SmartRF Studio 7 [57], a Windows application for evaluating radio for TIs' CC1xxx low-power RF devices. We configured the chip's registers to have $434 \mathrm{MHz}$ carrier frequency, $14 \mathrm{dBm}$ transmit power, $40 \mathrm{MHz}$ frequency for the active crystal, symbol rate of 1250 symbols per second (sps), and modulation format of 4Gaussian frequency-shift keying (4-GFSK) modulation format. The parameters of CC1200 is defined in Table III.

\section{Experimental Results}

As mentioned before, the designed wireless robotic system has the challenge of water environment, in which the signal should penetrate water to be realized correctly by the receiver. We set an experiment to measure the received signal strength (RSS) of the transmitted signal in our wireless system, and also the packet error (i.e. the number of packets that are received incorrectly). Packet errors are calculated as the ratio of the number of packets that are not received to all packets in the water environment. To evaluate RSS and packet error, a wireless sensor module is designed and submerged in a bucket of water (Fig. 19b). In this experiment, five sensors (FlexiForce sensors) are connected to five channels of the MCU. The dynamic range of the sensors is similar to the chemical sensors. The MCU is connected to the CC1200 evaluation module (Fig. 19a) and both are powered by a battery. In Table IV] the experiment parameters are listed. 


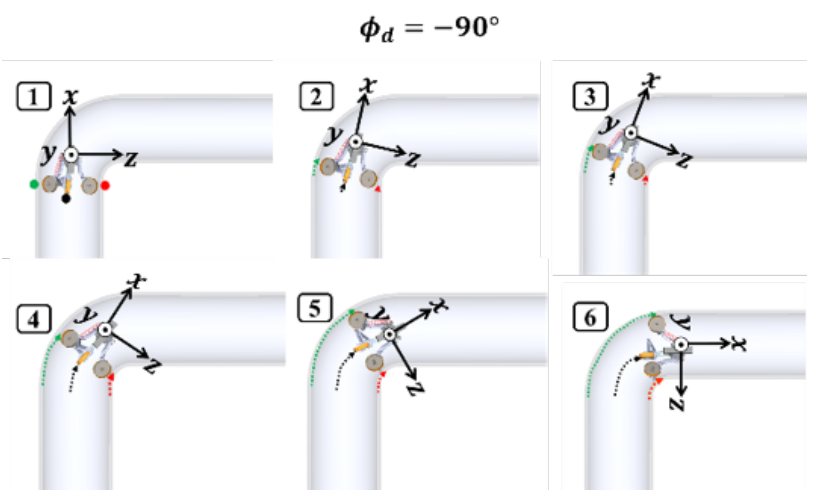

(a)

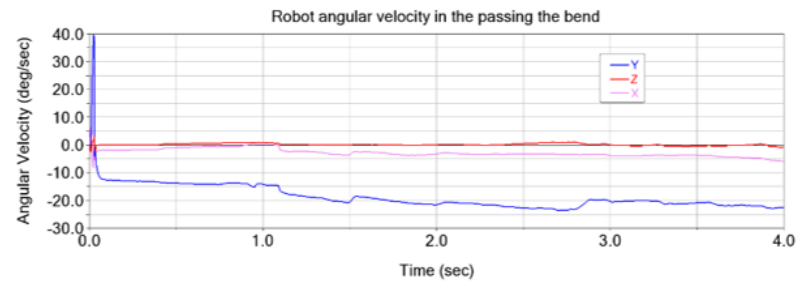

(b)

Fig. 16: The robot and the controller performance in a $90^{\circ}$ bend with a 12-inch diameter pipe with ADMAS MATLAB cosimulation. (a) The sequence of motion during passing through the bend. (b) The robot's angular velocities around the x-axis, y-axis, and z-axis.

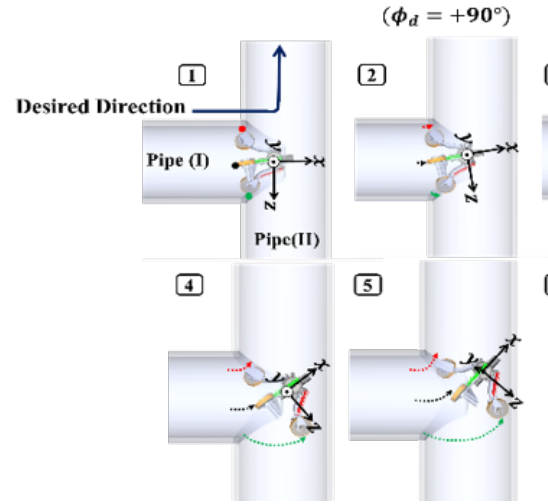

(a)

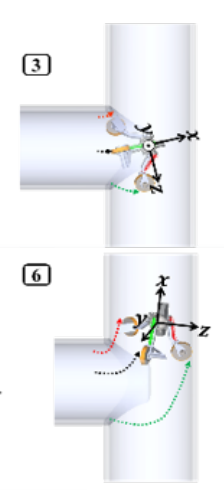

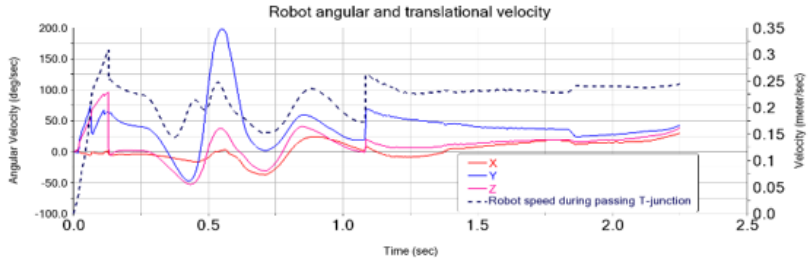

(b)

Fig. 17: Performance of the proposed phase 2 control in T-junctions with simulation with ADAMS MATLAB co-simulation. (a) Sequences of motion of the robot in T-junction. (b) The angular velocities of the robot around the $\mathrm{x}$-axis, $\mathrm{y}$-axis, and $\mathrm{z}$-axis and linear speed during rotation.

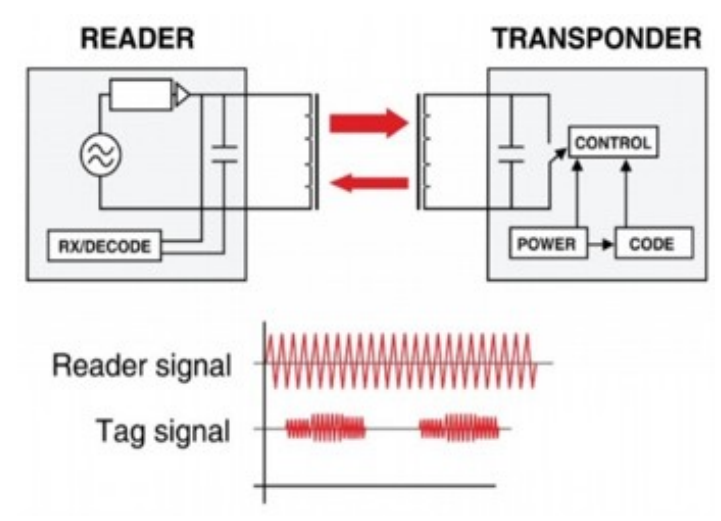

Fig. 18: A full-duplex (FDX) RFID module. The photo is adopted from [44].

The sensor module sends the sensor measurements, continuously. A transceiver (reader) module is located at a distance of around $10 \mathrm{~m}$ away from the sensor module that receives the data packets. A Graphical User Interface (GUI) (Fig. 19c) offered by SmartRF Studio is connected to the reader and
TABLE IV: EXPERIMENTAL RESULTS OF THE PROPOSED WIRELESS SENSOR MODULE IN WATER MEDIUM.

\begin{tabular}{|cc|}
\hline Parameter [unit] & Description \\
\hline \hline Preamble count (bytes) & 3 \\
Packet length (byte) & 13 \\
Transmit power (dBm) & 14 \\
Transmitter and receiver distance (m) & 10 \\
Received Signal Strength (dBm) & -61 \\
Packet error & 0 \\
\hline
\end{tabular}

shows the RSS of the data packets and bit and packet errors. The average RSS in our experiments is measured equal to $-61 \mathrm{dBm}$. Also, the bit and packet error was measured zero percent on the experiment. Hence the proposed system can penetrate the water medium and suitable for underground applications.

\section{SYSTEM IMPLEMENTATION}

The implementation of our robot consists of electrical and mechanical designs. The electrical hardware of the system 


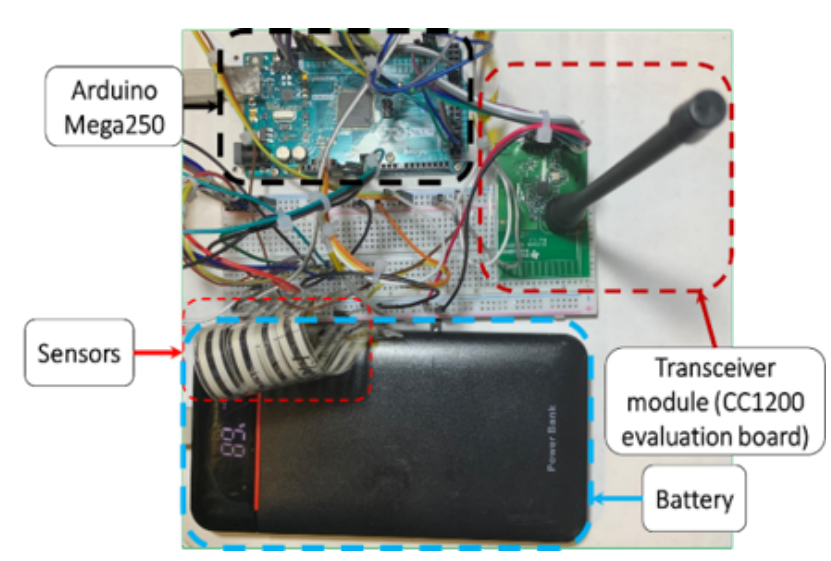

(a)

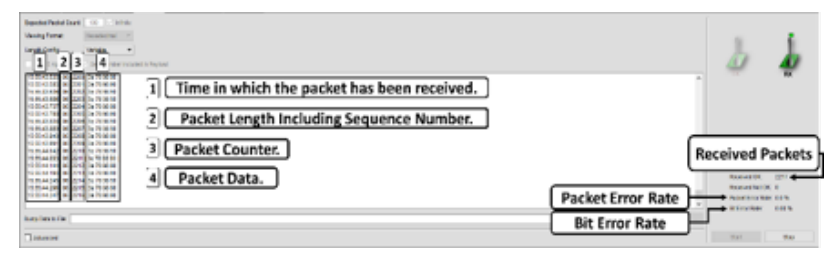

(c)

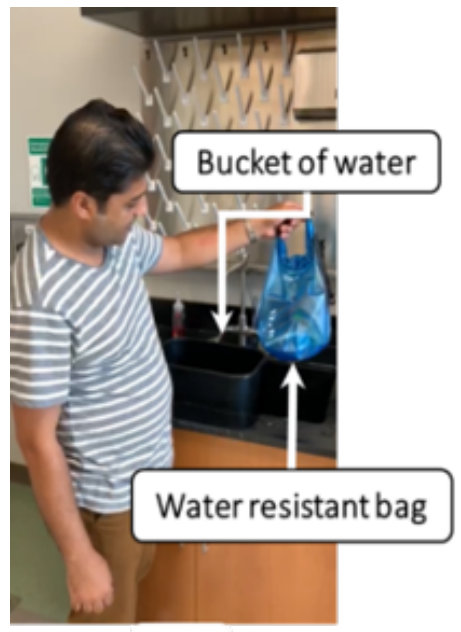

[1]

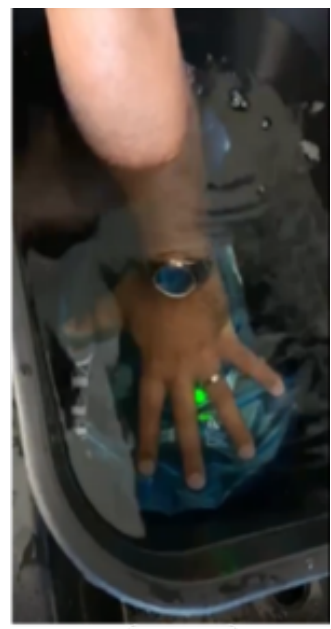

[2]

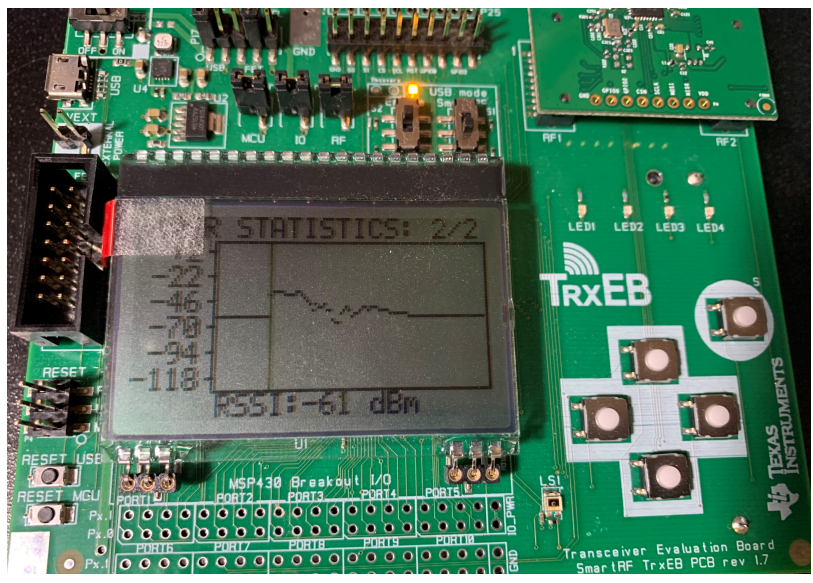

(d)

Fig. 19: Experiment setup. (a) Wireless sensor transmitter. (b) Experiment setup in bucket of water. (c) Graphical User Interface (GUI) that shows the packets, packet counters and packet and bit errors. (d) received signal strength (RSS) analyzer.

includes the power management circuit, analog drivers for the robot's actuators, analog sensors, and a low-power embedded system that controls the motion of the robot, acquires the sensors data, and provides the bidirectional data communication link with the base station.

\section{A. Electrical System}

1) Power Management: A $12 \mathrm{~V}$ lithium rechargeable battery with a capacity of 18A.h is the power source to the system. Regarding the analog and digital circuits as the main subcircuits of the electrical design, three DC voltage levels of $12 \mathrm{~V}, 5 \mathrm{~V}$, and $3.3 \mathrm{~V}$ need to be regulated and supplied. Therefore, while the battery voltage (VBAT) supplies the motor drives directly, it is stepped down to less than $6 \mathrm{~V}$ through a high-efficiency DC-DC buck converter (TPS62150, Texas Instrument -TI-), and then is regulated to $5 \mathrm{~V}$ and $3.3 \mathrm{~V}$ through a dual-channel low-noise low drop-out linear voltage regulator (TPS7A87, TI). The two-step voltage regulation provides efficient power consumption and besides, minimizes the dissipation as heat. The $5 \mathrm{~V}$ output voltage supplies analog sensors, the logic supply of the drivers, and the microcontroller. Also, the $3.3 \mathrm{~V}$ provides the power for the RF transceiver (i.e. CC1200) and the IMU (i.e. BMI160).

Current consumption corresponding to each of these three regulated DC voltages and amount of noise each of the digital, analog, and RF subcircuits generate, directly affect the design of the PCB of the electrical system, the number of layers, and independent islands for. In addition, isolated ground planes for the loads connected to the $12 \mathrm{~V}$ and for the subcircuits supplied by $5 \mathrm{~V}$ and $3.3 \mathrm{~V}$ are considered, too.

2) Microprocessor and Digital Circuit: A low-power microcontroller (ATmega2560, Atmel) is the heart of the embedded system. In fact, it controls the robot drivers, stablishes a serial peripheral interface (SPI) data communication with a the $\mathrm{CC} 1200$, and communicates with the IMU (i.e. BMI160) through an inter-integrated circuit 
(I2C) protocol. Furthermore, the MCU has an embedded analog-to-digital converter (ADC) which takes samples from four analog sensors with 10 bits resolution. The RF transceiver implements a bidirectional data communication link with the stationary units through the MCU to transmit sensors' digitized samples and receive robot motion control commands.

Due to incompatibility in the DC supply rating between the MCU (powered by $5 \mathrm{~V}$ ) and the RF transceiver and the IMU (powered by $3.3 \mathrm{~V}$ ), it was not possible to connect the SPI and I2C data buses between them. In this regard, the data lines are connected through digital buffer gates (SN74LVC2G34, TI). While the buffers accept input voltage of $5 \mathrm{~V}$, the output are limited to the DC power supply of the buffers, which are $3.3 \mathrm{~V}$.

3) The Robot Drivers and Analog Circuit: To drive three wings of our robot, two high-current full-bridge dual drivers (L298, STMicroelectronics) provide the electrical control for the motors. Control input lines to the drivers are controlled through a pulse-width modulation (PWM) provided by the MCU and hence, motors can be driven independently and simultaneously. Besides, each motor is equipped with an encoder which measures angular velocity of the motors as discussed earlier in this paper. Also, the system is equipped with a piezoelectric diaphragm micropump (mp6, Bartels Mikrotechnik) for pumping liquids inside our robot with varying flow rates controlled by a controller (mp6-OEM). In the design, we adopted for our application, frequency, and amplitude to the micropump are predefined by the internal circuit [58]. Furthermore, five analog sensors are added to the system to multi-parameter measurements for water quality monitoring. The Four sensors provide the output in terms of change voltage and the nature of the fifth one's output is current (i.e. miniaturized sensor for chlorine measurement in water [3]). In this regard, five analog buffers between the sensor's outputs and the ADC inputs of the microcontroller based on low-power operational amplifiers (OPA191, TI) are designed. Since the output values of four voltage-output sensors vary in the range of $-0.5 \mathrm{~V}$ to $0.5 \mathrm{~V}$, the analog buffers are designed such that the ADCs read values centered at half of its reference voltage.

The voltage-output sensors values are mapped to $1.25+0.75$ $V_{\text {sensor }}$ through a voltage divider and then, sampled by the ADCs. Therefore, $V_{\text {sensor }}$ should be extracted through $\left(V_{A D C}-1.25\right) / 0.75$. The output of single current-output sensor varies in the range of 0 to $50 \mathrm{~A}$ and so, needs to be converted to voltage through passing an appropriate resistor. The PCB antenna is a terminal antenna with a surface mount assembly (SMA) connection. It is designed for a frequency range of 433.05 434.79MHz and $0 \mathrm{dBi}$ gain from Taoglas Inc. [59]. In Fig. 20, the hardware architecture of the PCB and the connection of different components are shown.

\section{B. Mechanical System}

In Fig. 21 the designed PCB, and the components on it for the wireless communication setup, the control unit, the power management, the actuation unit, and the measurement

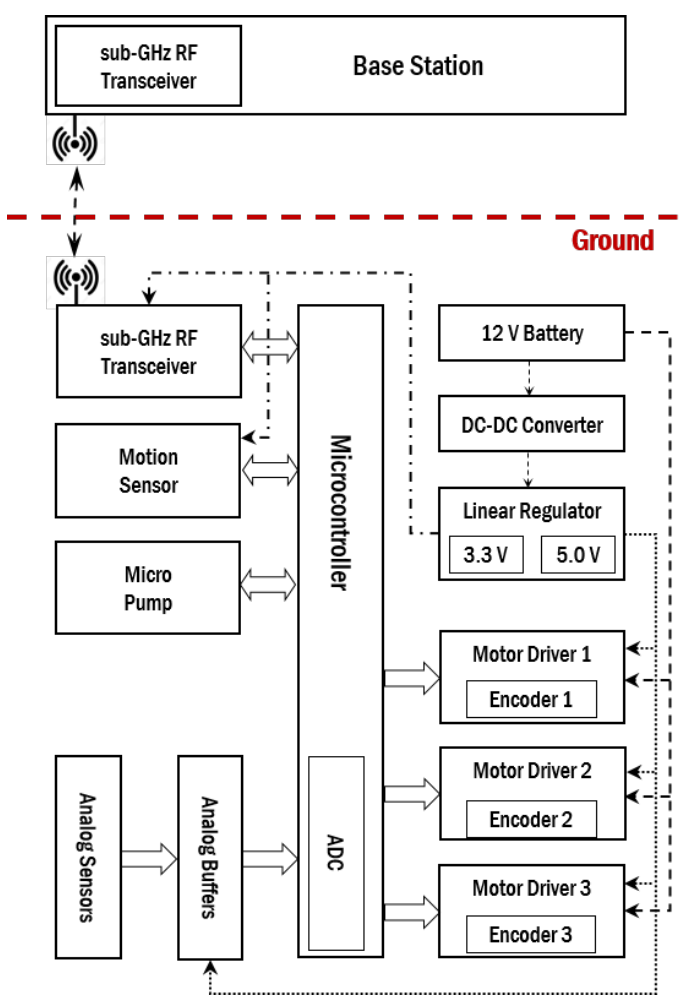

Fig. 20: The block diagram the printed circuit board (PCB) of SmartCralwer robot.

unit are shown. The PCB is located in the central processor (see [a] in Fig. 22). The diameter of the PCB is 2.94-inch which is located on the surface in the control part of the central processor (see [b] in Fig. 22). An area is designed on the central processor to make enough space for the motor drivers to facilitate heat transfer (see [b] in Fig. 22). Power from the battery is connected to the PCB with power socket through sealed hole on the central processor (see [b] in Fig. 22). Water circulates in sensor modules (if the robot is supposed to do water quality monitoring) through water inlets and outlets that are shown in [c] in Fig. 22. The exploded view of the central processor and different components in the central are shown in [d] in Fig. 22.

\section{CONCLUSION}

The in-pipe robot system we proposed in this research benefits from the characteristics of soft robots with the underactuated mechanism that provides high manoeuvrability for chattered environments, and dexterity of the traditional wheeled mobile robots. The two-phase controller enables a fast and stable motion in the highly pressurized environment of the water pipes. Switching between two phases of the controller enables smart navigation for the robot. The active radio frequency identification (RFID) that we proposed in this paper enables a reliable wireless communication for the robot (i.e. sensor measurement transmission) in the highly attenuative of underground environment. The experiment results showed a $10 \mathrm{~m}$ read range with $-61 \mathrm{dBm}$ received signal 
(1)

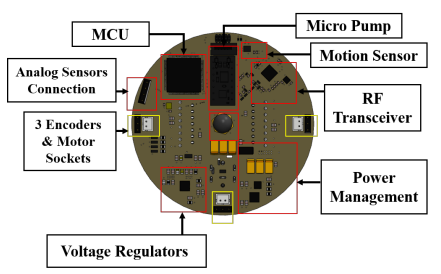

(2)

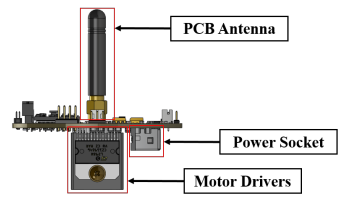

(3)

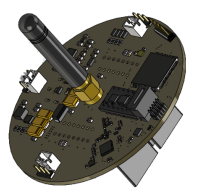

(4)

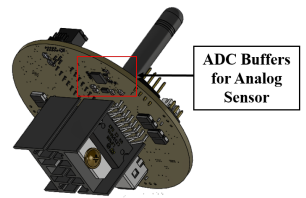

Fig. 21: Printed circuit board (PCB) for the robot and its components.
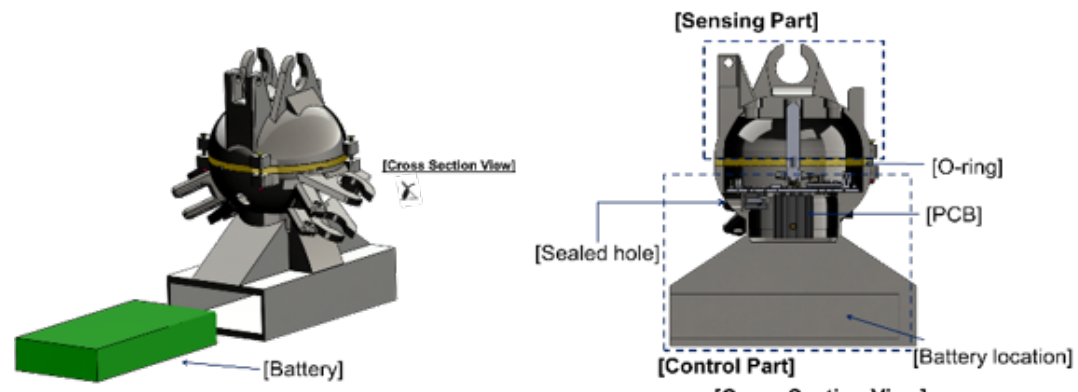

[a]

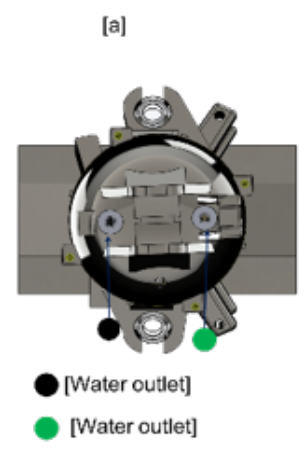

[c]

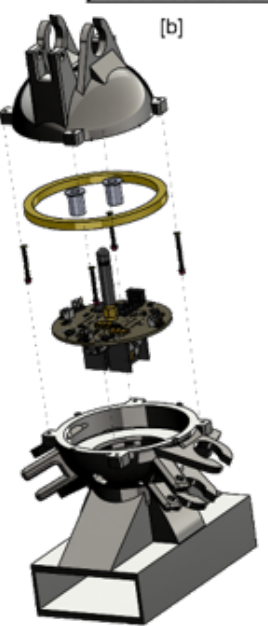

[d]

Fig. 22: The central processor in the SmartCrawler robot. a) Perspective view. b) Cross section view, c) Water inlet and outlet on the central processor. d) Exploded view of the central processor.

strength (RSSI). The combination of the robotic sensor, multiphase controller, and the wireless communication system suggests promising system for water quality monitoring and leak detection in large pipes of water distribution systems (WDS).

In our future work, we will develop a firmware that facilitates wireless control and data transmission during operation for the SmartCrawler. The wireless control is our solution for smart navigation for in-pipe robot; a challenging and untouched task in this field.

\section{REFERENCES}

[1] A. L. Vickers, "The future of water conservation: Challenges ahead," Journal of Contemporary Water Research and Education, vol. 114, no. 1, p. 8, 1999.

[2] E. Canada, "Threats to water availability in canada," 2004.
[3] R. Wu, "Development of a mobile sensor for potable water quality monitoring," Ph.D. dissertation, Purdue University, 2014.

[4] B. H. Lee and R. A. Deininger, "Optimal locations of monitoring stations in water distribution system," Journal of Environmental Engineering, vol. 118, no. 1, pp. 4-16, 1992.

[5] D. Chatzigeorgiou, K. Youcef-Toumi, and R. Ben-Mansour, "Design of a novel in-pipe reliable leak detector," IEEE/ASME Transactions on mechatronics, vol. 20, no. 2, pp. 824-833, 2014.

[6] F.-L. Chen, B.-C. Yang, S.-Y. Peng, and T.-C. Lin, "Applying a deployment strategy and data analysis model for water quality continuous monitoring and management," International Journal of Distributed Sensor Networks, vol. 16, no. 6, p. 1550147720929825, 2020.

[7] R. Fletcher and M. Chandrasekaran, "Smartball: a new approach in pipeline leak detection," in International Pipeline Conference, vol. 48586, 2008, pp. 117-133.

[8] R. Wu, W. Salim, S. Malhotra, A. Brovont, J. Park, S. Pekarek, M. Banks, and D. Porterfield, "Self-powered mobile sensor for inpipe potable water quality monitoring," in Proceedings of the 17th International Conference on Miniaturized Systems for Chemistry and Life Sciences, 2013, pp. 14-16.

[9] L. Perelman and A. Ostfeld, "Operation of remote mobile sensors for 
security of drinking water distribution systems," Water research, vol. 47, no. 13, pp. 4217-4226, 2013.

[10] H. Sato, Y. Mano, F. Ito, T. Yasui, M. Okui, R. Nishihama, and T. Nakamura, "Proposal for pipeline-shape measurement method based on highly accurate pipeline length measurement by imu sensor using peristaltic motion characteristics," in 2020 IEEE/ASME International Conference on Advanced Intelligent Mechatronics (AIM). IEEE, 2020, pp. 874-881.

[11] K. Miyasaka, G. Kawano, and H. Tsukagoshi, "Long-mover: Flexible tube in-pipe inspection robot for long distance and complex piping," in 2018 IEEE/ASME International Conference on Advanced Intelligent Mechatronics (AIM). IEEE, 2018, pp. 1075-1080.

[12] M. Kamata, S. Yamazaki, Y. Tanise, Y. Yamada, and T. Nakamura, "Morphological change in peristaltic crawling motion of a narrow pipe inspection robot inspired by earthworms locomotion," Advanced Robotics, vol. 32, no. 7, pp. 386-397, 2018.

[13] Y. Qu, P. Durdevic, and Z. Yang, "Smart-spider: Autonomous self-driven in-line robot for versatile pipeline inspection," Ifac-papersonline, vol. 51, no. 8, pp. 251-256, 2018.

[14] Y.-S. Kwon and B.-J. Yi, "Development of a pipeline inspection robot system with diameter of $40 \mathrm{~mm}$ to $70 \mathrm{~mm}$ (tbot-40)," in 2010 IEEE International Conference on Mechatronics and Automation. Ieee, 2010 , pp. 258-263.

[15] A. V. S. Bhadoriya, V. K. Gupta, and S. Mukherjee, "Development of in-pipe inspection robot," Materials Today: Proceedings, vol. 5, no. 9, pp. 20769-20776, 2018.

[16] M. Abdellatif, H. Mohamed, M. Hesham, A. Abdelmoneim, A. Kamal, and A. Khaled, "Mechatronics design of an autonomous pipe-inspection robot," in MATEC Web of Conferences, vol. 153. EDP Sciences, 2018, p. 02002.

[17] G. H. Mills, J. H. Liu, B. Y. Kaddouh, A. E. Jackson, and R. C. Richardson, "Miniature magnetic robots for in-pipe locomotion," in Robotics Transforming the Future: Proceedings of CLAWAR 2018: The 21 st International Conference on Climbing and Walking Robots and the Support Technologies for Mobile Machines. CLAWAR Association Ltd, 2018, pp. 289-300.

[18] A. H. Sheikh, F. A. Mir, and S. Hussain, "Pipe inspection robot," 2018.

[19] M. A. A. Wahed and M. R. Arshad, "Wall-press type pipe inspection robot," in 2017 IEEE 2nd International Conference on Automatic Control and Intelligent Systems (I2CACIS). IEEE, 2017, pp. 185-190.

[20] Q. Liu, T. Ren, and Y. Chen, "Characteristic analysis of a novel in-pipe driving robot," Mechatronics, vol. 23, no. 4, pp. 419-428, 2013.

[21] Y.-S. Kwon and B.-J. Yi, "Design and motion planning of a two-module collaborative indoor pipeline inspection robot," IEEE Transactions on Robotics, vol. 28, no. 3, pp. 681-696, 2012.

[22] J.-y. Nagase and F. Fukunaga, "Development of a novel crawler mechanism for pipe inspection," in IECON 2016-42nd Annual Conference of the IEEE Industrial Electronics Society. IEEE, 2016, pp. 5873-5878.

[23] R. Tao, Y. Chen, and L. Qingyou, "A helical drive in-pipe robot based on compound planetary gearing," Advanced Robotics, vol. 28, no. 17, pp. 1165-1175, 2014.

[24] A. Singh, E. Sachdeva, A. Sarkar, and K. M. Krishna, "Design and optimal springs stiffness estimation of a modular omnicrawler in-pipe climbing robot," arXiv preprint arXiv:1706.06418, 2017.

[25] A. Hadi, A. Hassani, K. Alipour, R. Askari Moghadam, and P. Pourakbarian Niaz, "Developing an adaptable pipe inspection robot using shape memory alloy actuators," Journal of Intelligent Material Systems and Structures, vol. 31, no. 4, pp. 632-647, 2020.

[26] D. Lee, J. Park, D. Hyun, G. Yook, and H.-s. Yang, "Novel mechanisms and simple locomotion strategies for an in-pipe robot that can inspect various pipe types," Mechanism and Machine Theory, vol. 56, pp. 52-68, 2012.

[27] A. C. Yunus, Fluid Mechanics: Fundamentals And Applications (Si Units). Tata McGraw Hill Education Private Limited, 2010.

[28] L. Shao, Y. Wang, B. Guo, and X. Chen, "A review over state of the art of in-pipe robot," in 2015 IEEE International Conference on Mechatronics and Automation (ICMA). IEEE, 2015, pp. 2180-2185.

[29] I. F. Akyildiz and E. P. Stuntebeck, "Wireless underground sensor networks: Research challenges," Ad Hoc Networks, vol. 4, no. 6, pp. 669-686, 2006.

[30] D. R. Frankl, Electromagnetic theory. Prentice Hall, 1986.

[31] Z. Sun and I. F. Akyildiz, "Underground wireless communication using magnetic induction," in 2009 IEEE International Conference on Communications. IEEE, 2009, pp. 1-5.

[32] X. Tan, Z. Sun, and I. F. Akyildiz, "Wireless underground sensor networks: Mi-based communication systems for underground applications."
IEEE Antennas and Propagation Magazine, vol. 57, no. 4, pp. 74-87, 2015.

[33] S. Kazeminasab, M. Aghashahi, R. Wu, and M. K. Banks, "Localization techniques for in-pipe robots in water distribution systems," in 2020 8th International Conference on Control, Mechatronics and Automation (ICCMA), 2020, pp. 6-11.

[34] N. Ahmed, J. Hoyt, A. Radchenko, D. Pommerenke, and Y. R. Zheng, "A multi-coil magneto-inductive transceiver for low-cost wireless sensor networks," in 2014 Underwater Communications and Networking (UComms). IEEE, 2014, pp. 1-5.

[35] D. Wu, D. Chatzigeorgiou, K. Youcef-Toumi, and R. Ben-Mansour, "Node localization in robotic sensor networks for pipeline inspection," IEEE Transactions on Industrial Informatics, vol. 12, no. 2, pp. 809819, 2015.

[36] S. Kazeminasab, R. Jafari, and M. K. Banks, "An lqr-assisted control algorithm for an under-actuated in-pipe robot in water distribution systems," in The 36th ACM/SIGAPP Symposium On Applied Computing, 2021, in press.

[37] CSIR, "Guidelines for human settlement planning and design." 2005.

[38] S. Kazeminasab, A. Akbari, R. Jafari, and M. K. Banks, "Design, characterization, and control of a size adaptable in-pipe robot for water distribution systems," in 22nd IEEE International Conference on Industrial Technology, 2021, in press.

[39] S. Kazeminasab, M. Aghashahi, and M. K. Banks, "Development of an inline robot for water quality monitoring," in 2020 5th International Conference on Robotics and Automation Engineering (ICRAE), 2020, pp. 106-113.

[40] J. P. Hespanha, Linear systems theory. Princeton university press, 2018.

[41] V. Klemm, A. Morra, C. Salzmann, F. Tschopp, K. Bodie, L. Gulich, N. Küng, D. Mannhart, C. Pfister, M. Vierneisel et al., "Ascento: A twowheeled jumping robot," in 2019 International Conference on Robotics and Automation (ICRA). IEEE, 2019, pp. 7515-7521.

[42] Y. Wu, D. Chatzigeorgiou, K. Youcef-Toumi, and M. Zribi, "Modeling and parameter estimation for in-pipe swimming robots," in 2015 American Control Conference (ACC). IEEE, 2015, pp. 2007-2013.

[43] R. Mahony, T. Hamel, and J.-M. Pflimlin, "Nonlinear complementary filters on the special orthogonal group," IEEE Transactions on automatic control, vol. 53, no. 5, pp. 1203-1218, 2008.

[44] K. Sattlegger and U. Denk, "Navigating your way through the rfid jungle," White paper, Texas Instruments, 2014.

[45] T. Knowles, J. Larmouth, and K. G. Knightson, Standards for open systems interconnection. BSP Professional Books, 1987, vol. 3.

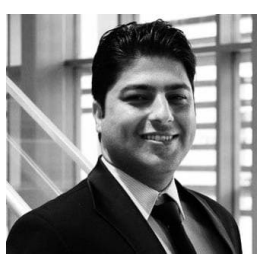

Saber Kazeminasab (S'18) is a PhD student in the Department of Electrical and Computer Engineering, Texas A\&M University, College Station, TX, USA. He received the B.Sc. degree from the Iran University of Science and Technology, Tehran, Iran, in 2014, in mechanical engineering, and the M.Sc. degree from the University of Tehran, Tehran, Iran, in 2017 in mechatronics engineering.

His research interests include mechatronics, and actuator design. robotics, control theories, mechanism design, 


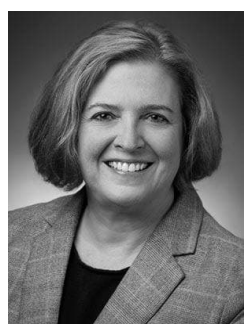

M. Katherine Banks is a professor of Civil engineering department and currently Vice Chancellor of Engineering for The Texas A\&M University System and Dean of the Texas A\&M University College of Engineering. She is an Elected Fellow of the American Society of Civil Engineers, was elected in 2014 to the National Academy of Engineering, and was formerly the Jack and Kay Hockema Professor at Purdue University. Her research interests include applied microbial systems, biofilm processes, wastewater treatment and reuse, and phytoremediation bioremediation. She received her Ph.D. in 1989 from Duke University.

At Texas A\&M, she helped establish the EnMed program (led by Roderic Pettigrew, Ph.D., M.D.), an innovative engineering medical school option created by Texas A\&M University and Houston Methodist Hospital, designed to educate a new kind of physician who will create transformational technology for health care.received her Bachelor of Science in Engineering from the University of Florida, Master of Science in Engineering from the University of North Carolina, and Doctorate of Philosophy in civil and environmental engineering from Duke University.Banks is the recipient of the American Society of Civil Engineers Petersen Outstanding Woman of the Year Award, American Society of Civil Engineers Rudolph Hering Medal, Purdue Faculty Scholar Award, Sloan Foundation Mentoring Fellowship and the American Association of University Women Fellowship.On February 13, 2019, she was named to the Board of Directors of Halliburton. 